\title{
Extremal Divisors on Moduli Spaces of Rational Curves with Marked Points
}

\author{
Morgan OPIE
}

\begin{abstract}
We study effective divisors on $\bar{M}_{0, n}$, focusing on hypertree divisors introduced by Castravet and Tevelev and on the proper transforms of divisors on $\bar{M}_{1, n-2}$ introduced by Chen and Coskun. We relate these two types of divisors and exhibit divisors on $\bar{M}_{0, n}$ for $n \geq 7$ that furnish counterexamples to a conjectural description of the effective cone of $\bar{M}_{0, n}$ given by Castravet and Tevelev.
\end{abstract}

\section{Introduction}

The moduli space $M_{0, n}$ parameterizes equivalence classes of $n$ distinct marked points on $\mathbb{P}^{1}$ under the action of $P G L_{2}$. We will be primarily concerned with $\bar{M}_{0, n}$, the Deligne-Mumford compactification of $M_{0, n}$ by stable rational curves with $n$ marked points. The Deligne-Mumford compactification parameterizes nodal trees of $\mathbb{P}^{1} \mathrm{~s}$ with $n$ markings such that each component has at least three "special" points (markings or nodes) modulo automorphisms (see Figure 1).

The locus $\bar{M}_{0, n} \backslash M_{0, n}$ is a union of boundary divisors, defined as follows: for $I \subset\{1, \ldots, n\}$ with both $I$ and $\{1, \ldots, n\} \backslash I$ of size at least two, the boundary divisor $\delta_{I}$ consists of classes of stable rational curves in $\bar{M}_{0, n} \backslash M_{0, n}$ with a node separating the markings corresponding to indices in $I$ and $\{1, \ldots, n\} \backslash I$.

Significantly, $\bar{M}_{0, n}$ can be realized as an iterated blow-up of $\mathbb{P}^{n-3}$ via a Kapranov morphism. Any Kapranov morphism restricts to an isomorphism of $M_{0, n}$ with its image, and any boundary divisor is contracted by some Kapranov morphism. Hence, each boundary divisor generates an extremal ray of the effective cone of $\bar{M}_{0, n}$, and select boundary divisors together with the pull-back of a hyperplane class under a Kapranov morphism comprise free generators for the class group $\mathrm{Cl}\left(\bar{M}_{0, n}\right)[\mathrm{K}]$. We will use these Kapranov generators throughout the paper.

In Section 2, we describe a method of specifying divisors on $\bar{M}_{0, n}$ via polynomials in $n$ variables. We discuss how to compute the classes of these divisors and include Macaulay 2 code to compute classes. Although useful for checking results on $\bar{M}_{0, n}$ with $n \leq 10$, the code is not practical for large $n$.

In Section 3, we recall the definitions of hypertrees and hypertree divisors from $[\mathrm{CT}]$. A major result of $[\mathrm{CT}]$ is that hypertree divisors corresponding to "irreducible" hypertrees are exceptional divisors of some birational contraction and

Received June 26, 2014. Revision received March 7, 2016.

This research was supported by NSF grant DMS-1001344 (PI Jenia Tevelev). 

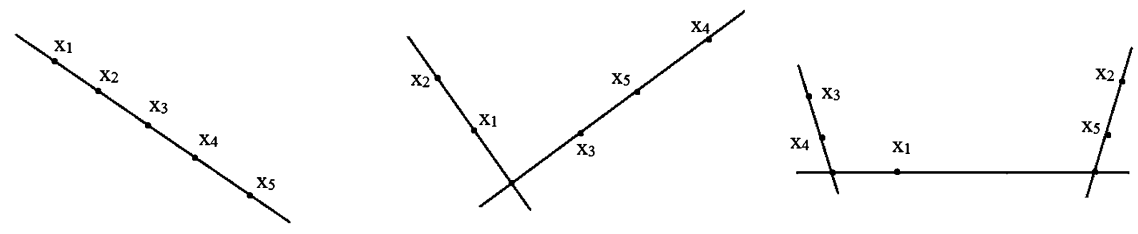

Figure 1 Examples of stable rational curves, $n=5$

hence generate extremal rays of the effective cone of $\bar{M}_{0, n}$. In [CT], it is further speculated that:

CONJECTURE 1.1. The effective cone of $\bar{M}_{0, n}$ is generated by boundary divisors and by divisors parameterized by irreducible hypertrees and the pull-backs of these divisors under forgetful morphisms.

This motivates us to study hypertree divisors and their classes. We generalize a result of [CT] to obtain polynomials specifying all hypertree divisors and use our Macaulay2 program to compute all irreducible hypertree divisor classes on $\bar{M}_{0, n}$ for $6 \leq n \leq 10$. We then turn our attention to other effective divisors.

Chen and Coskun [CC] construct divisors on $\bar{M}_{1, n}$ using $n$-tuples $\left(a_{1}, \ldots, a_{n}\right)$ of integers such that $\sum a_{i}=0$. They show that if $\operatorname{gcd}\left(a_{1}, \ldots, a_{n}\right)=1$, then the divisor corresponding to the $n$-tuple is a rigid, extremal effective divisor. We examine the proper transforms of these divisors on $\bar{M}_{0, n+2}$ with respect to the clutching morphism that glues the two markings (we call these proper transforms Chen-Coskun divisors). We first find formulas for the classes of Chen-Coskun divisors and then prove results relating Chen and Coskun and hypertree divisors. In particular, we show that the Chen-Coskun divisor associated to the $n$-tuple $(1,1, \ldots,-1,-1, \ldots)$ coincides with a particular hypertree divisor.

Next, we investigate the extremality of Chen-Coskun divisors. Such divisors need not be extremal, as examples in Section 7 show. However, in Section 5, we show that the Chen-Coskun divisor corresponding to $(n, 1,-1,-1,-1, \ldots)$ is always nonboundary extremal. Moreover, these particular Chen-Coskun divisors are neither hypertree divisors nor pull-backs of hypertree divisors. Hence, they furnish counterexamples to Conjecture 1.1.

In Section 6, we give a proof of a well-known criterion for extremality used in Section 5. In fact, we show that our criterion not only guarantees the extremality, but also that the effective cone is "not rounded" near the given divisor. No reference for this fact was found.

In Section 7, we further investigate the extremality and rigidity of Chen and Coskun classes. We give criteria for rigidity and nonextremality via conditions on the $n$-tuple defining a Chen-Coskun divisor and discuss implications for constructing "large" families of extremal divisors on $\bar{M}_{0, n}$. 


\section{Divisors on $\bar{M}_{0, n}$ Specified by Polynomials}

The following diagram is useful in studying divisors on $\bar{M}_{0, n}$ :

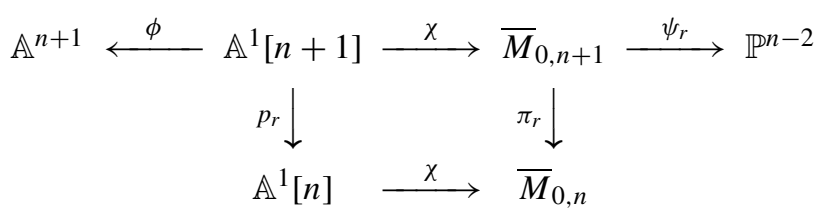

Here $\psi_{r}$ is the Kapranov morphism in index $r$. A Kapranov morphism $\psi_{r}: \bar{M}_{0, k} \rightarrow \mathbb{P}^{k-3}$ for $1 \leq i \leq n$ is constructed by fixing $k-1$ points in general position in $\mathbb{P}^{k-3}$ and labeling the points $p_{t}$ for $t \in\{1, \ldots, k\} \backslash\{r\}[\mathrm{K} 1]$. The relevant fact for our purposes is that given $I \subset\{1, \ldots, k\} \backslash\{r\}$, the image of $\delta_{I \cup\{r\}}$ under $\psi_{r}$ is the linear span $\left\langle p_{t}\right\rangle_{t \in I}$. For $|I| \leq k-4, \psi_{r}$ contracts the divisor $\delta_{I \cup\{r\}}$; these are the only exceptional divisors of $\psi_{r}$. This gives the choice of free generators for $\mathrm{Cl}\left(\bar{M}_{0, k}\right)$ discussed in Section 1, namely the classes of boundary divisors $E_{I}^{r}:=\delta_{I \cup\{r\}}$ for $1 \leq|I| \leq k-4$ and of $H=\psi_{r}^{-1}(h)$ for $h$ a hyperplane in $\mathbb{P}^{n-3}$. We refer to the free generating set $\left\langle E_{I}^{r}, H\right\rangle$ obtained via the map $\psi_{r}$ as the Kapranov basis in index $r$, index $r$ Kapranov basis, or $r$ th Kapranov basis. When the "special" index is clear, we omit the superscript.

The map $\pi_{r}$ is the forgetful morphism in index $r$ : drop the $r$ th marking on a stable rational curve and stabilize if necessary.

The space $\mathbb{A}^{1}[n+1]$ is Fulton-MacPherson configuration space over $\mathbb{A}^{1}$, a partial compactification of the space parameterizing $n+1$ distinct marked points on $\mathbb{A}^{1}$. The map $\phi$ is an iterated blow-up of $\mathbb{A}^{n+1}$ along partial diagonals that defines $\mathbb{A}^{1}[n+1]$. This gives a basis of $\mathrm{Cl}\left(\mathbb{A}^{1}[n+1]\right)$ comprised of exceptional divisors $\Delta_{I}$ over partial diagonals $\operatorname{Diag}_{I}:=\left\{x_{i}=x_{j} \mid i, j \in I\right\}$ for $3 \leq|I| \leq n+1$ [FM]. A general element of an exceptional divisor $\Delta_{I}$ consists of a copy of $\mathbb{A}^{1}$ containing marked points in $\{1, \ldots, n\} \backslash I$, with a nodal tree of $\mathbb{P}^{1} \mathrm{~s}$ containing the marked points in $I$ attached.

As discussed in [FM, p. 195], we have a map $p_{r}: \mathbb{A}^{1}[n+1] \rightarrow \mathbb{A}^{1}[n]$ that drops the $r$ th marking on an element of $\mathbb{A}^{1}[n+1]$ (analogous to the forgetful map $\left.\pi_{r}: \bar{M}_{0, n+1} \rightarrow \bar{M}_{0, n}\right)$. Moreover, we have a map from $\mathbb{A}^{1}[n+1]$ to $\mathbb{P}^{1}[n+1]$ : choose an embedding of $\mathbb{A}^{1}$ into $\mathbb{P}^{1}$ as an affine chart, and this induces a map taking an element of $\mathbb{A}^{1}[n+1]$ to a nodal tree of $\mathbb{P}^{1}$ s. Moreover, we have a map from $\mathbb{P}^{1}[n+1]$ into $\bar{M}_{0, n+1}$, mapping a tree of $\mathbb{P}^{1} \mathrm{~s}$ to its equivalence class modulo automorphisms. A slight obstruction arises because a tree of $\mathbb{P}^{1} \operatorname{s}$ in $\mathbb{P}^{1}[n+1]$ may not be stable, but this is easily resolved by stabilization. Composition gives the map $\chi$ on the diagram (1). The commutativity of the middle rectangle is evident from definitions.

Our goal in this section is to relate divisor classes in the class group of the Fulton-MacPherson space to those in the class group of the moduli space of stable rational curves with marked points. To this end, we compute the class of the pull-backs of boundary divisors from $\bar{M}_{0, k}$ under $\chi$. Note that the only boundary 
divisors contained in $\chi^{-1}\left(\delta_{I}\right)$ are $\Delta_{I}$ and $\Delta_{I^{c}}$. Hence, we have that

$$
\chi^{-1}\left(\delta_{I}\right) \sim m_{1} \Delta_{I}+m_{2} \Delta_{I^{c}} .
$$

It is well known that $m_{1}=m_{2}=1$, which is easy to prove by induction on $n$ :

LEMma 2.1. With maps and definitions as before, $\chi^{-1}\left(\delta_{I}\right) \sim \Delta_{I}+\Delta_{I^{c}}$.

We now return to the setup of (1). Given a prime, nonboundary divisor $D \subset$ $\bar{M}_{0, n}$, the divisor $\chi^{-1}(D)$ is irreducible: $\chi$ has irreducible fibers over $M_{0, n}$, and $\chi^{-1}(D)=\overline{\chi^{-1}\left(D \cap M_{0, n}\right)}$. Moreover, $\chi^{-1}(D)$ is not an exceptional divisor of $\phi$ since $D$ is nonboundary. Hence, $\chi^{-1}(D)$ is precisely $\phi_{*}^{-1}\left(\phi\left(\chi^{-1}(D)\right)\right)$, the proper transform of $\phi\left(\chi^{-1}(D)\right)$ with respect to $\phi$.

The fact that $\phi\left(\chi^{-1}(D)\right)$ is irreducible follows from irreducibility of $\chi^{-1}(D)$, so $\phi\left(\chi^{-1}(D)\right)=V(f)$ for some irreducible polynomial $f \in k\left[x_{1}, \ldots, x_{n}\right]$. In this case, we say that the divisor $D$ is specified by the polynomial $f$.

Using that $\mathbb{A}^{1}[n]$ is a blow-up of $\mathbb{A}^{n}$ along partial diagonals, we have that $\phi_{*}^{-1}(V(f)) \sim-\sum k_{J} \Delta_{J}$, where $k_{J}$ is the multiplicity of $f$ along the partial di$\operatorname{agonal}_{\operatorname{Diag}}:=\left\{x_{i}=x_{j} \mid i, j \in J\right\}$ for $|J| \geq 3$. The next results relate these multiplicities, which are easily computed when $f$ is known, to the class of $D$ with respect to Kapranov bases.

Theorem 2.2. Let $\pi_{n+1}: \bar{M}_{0, n+1} \rightarrow \bar{M}_{0, n}$ denote the forgetful morphism in index $n+1$. Given an irreducible polynomial $f \in k\left[x_{1}, \ldots, x_{n}\right]$ specifying a divisor $D$ on $\bar{M}_{0, n}$ as described before, we have that

$$
\pi_{n+1}^{-1}(D) \sim d H-\sum_{\substack{I \subset\{1, \ldots, n\} \\ 1 \leq|I| \leq n-3}} m_{I} E_{I}^{n+1},
$$

where $d=\operatorname{deg}(f)$, and $m_{I}$ is the multiplicity off along the complementary partial diagonal $\operatorname{Diag}_{I^{c}}$.

REMARK 2.3. We compute the class of $\pi_{n+1}^{-1}(D) \subset \bar{M}_{0, n+1}$ with respect to the $n+1$ Kapranov basis to preserve symmetry. Note that if $f \in k\left[x_{1}, \ldots, x_{n}\right]$ specifies $D$, then the same polynomial viewed as an element of $k\left[x_{1}, \ldots, x_{n+1}\right]$ specifies $\pi_{n+1}^{-1}(D)$. In Proposition 2.4, we explain how to convert the class of $\pi_{n+1}^{-1}(D) \subset \bar{M}_{0, n+1}$ to the class of $D \subset \bar{M}_{0, n}$ with respect to the index $r$ Kapranov basis.

Proof of Theorem 2.2. Define $N=\{1, \ldots, n\}$ and $N_{2}=\{1, \ldots, n-2\}$. Take $H$ as the pull-back of the linear span $\left\langle p_{i} \mid i \in N_{2}\right\rangle$ under $\psi_{n+1}$. Throughout this proof, we let $E_{I}=E_{I}^{n+1}$. Using our free generators $\left\langle E_{I}, H\right\rangle$ for $\mathrm{Cl}\left(\bar{M}_{0, n+1}\right)$ and $\left\langle\Delta_{I}\right\rangle$ for $\mathbb{A}^{1}[n+1]$, Lemma 2.1 implies that

$$
\chi^{-1}\left(E_{I}\right) \sim \Delta_{I \cup\{n+1\}}+\Delta_{N-I} .
$$


By [KT, 3.4],

$$
\begin{aligned}
\chi^{-1}(H) & \sim \sum_{\emptyset \neq J \subset N_{2}} \chi^{-1}\left(\delta_{J \cup\{n+1\}}\right) \\
& \sim \sum_{\emptyset \neq J \subset N_{2}}\left(\Delta_{J \cup\{n+1\}}+\Delta_{N-J}\right) \\
= & \sum_{J \subset N_{2},|J|>1} \Delta_{J \cup\{n+1\}}+\sum_{J \subsetneq N_{2},|J| \geq 1} \Delta_{N-J} \\
& +\sum_{i \in N_{2}} \Delta_{\{i, n+1\}}+\Delta_{\{n-1, n\}} .
\end{aligned}
$$

In (4), the last terms are those involving divisor classes over partial diagonals of codimension 1 in $\mathbb{A}^{n+1}$. These classes must be expressed in terms of our free generators. Using the relation

$$
\Delta_{\{\alpha, \beta\}} \sim-\sum_{\{\alpha, \beta\} \subsetneq I} \Delta_{I}
$$

which follows from [FM, p. 184], we see that

$$
\Delta_{\{a, n+1\}} \sim-\sum_{a \subsetneq J \subset N} \Delta_{J \cup\{n+1\}}
$$

and

$$
\Delta_{\{n-1, n\}} \sim-\sum_{\{n-1, n\} \subsetneq I} \Delta_{I} .
$$

Substituting these into (4) yields

$$
\begin{aligned}
\beta^{-1}(H) & \sim \sum_{J \subsetneq N_{2},|J| \geq 1} \Delta_{N-J}+\Delta_{n-1, n}+\Omega \\
& \sim \sum_{J \subsetneq N_{2},|J| \geq 1} \Delta_{N-J}-\sum_{\{n-1, n\} \subsetneq I \subset N} \Delta_{I}+\Omega,
\end{aligned}
$$

where $\Omega$ denotes a sum of free generators $\Delta_{I}$ with $n+1 \in I$. We subsequently redefine $\Omega$ to absorb such terms, which turn out to be superfluous. Summing over $N \backslash J$ for $J \subsetneq N_{2}$ and $|J| \geq 1$ is equivalent to summing over $I \subsetneq N$ with $\{n-1, n\} \subsetneq I$. Returning to (7), we obtain

$$
\beta^{-1}(H)=\sum_{\{n-1, n\} \subsetneq I \subsetneq N} \Delta_{I}-\sum_{\{n-1, n\} \subsetneq I \subset N} \Delta_{I}+\Omega=-\Delta_{N}+\Omega .
$$

We can now compute the class $\beta^{-1}(D)$ :

$$
\begin{aligned}
\beta^{-1}(D) & \sim \beta^{-1}\left(d H-\sum_{\substack{I \subset N \\
1 \leq|I| \leq n-3}} m_{I} E_{I}\right) \\
& \sim-d \Delta_{N}-\sum_{\substack{I \subset N \\
1 \leq|I| \leq n-3}} m_{I}\left(\Delta_{I \cup\{n+1\}}+\Delta_{N-I}\right)+\Omega
\end{aligned}
$$




$$
\beta^{-1}(D) \sim-d \Delta_{N}-\sum_{\substack{I \subset N \\ 1<|I|<n-2}} m_{I} \Delta_{N-I}+\Omega .
$$

For $I \subset N$ satisfying $2 \leq|I| \leq n-3$, we have a single term in (8) involving the free generator $\Delta_{N-I}$ with coefficient $m_{I}$. Hence, $m_{I}=k_{N-I}$.

It remains to determine the coefficient of $H$. The previous analysis shows that we have a single summand $d \Delta_{N}$ in the class of $\beta^{-1}(H)$, and the proper transforms of boundaries contribute no multiples of $\Delta_{N}$ to the sum. Hence, the multiplicity of $f$ along the diagonal $\operatorname{Diag}_{N}$ is $d$. We claim that if $D \subset \bar{M}_{0, n+1}$ is an irreducible nonboundary divisor and $f \in k\left[x_{1}, \ldots, x_{n}\right]$ satisfies $V(f)=\phi\left(\chi^{-1}(D)\right)$, then $f$ is a homogeneous polynomial. Furthermore, for some $g \in k\left[x_{1}, \ldots, x_{n}\right]$, we have that

$$
f\left(x_{1}, \ldots, x_{n}\right)=g\left(x_{1}-x_{2}, x_{2}-x_{3}, \ldots, x_{n-1}-x_{n}\right) .
$$

This follows from the fact that $V(f) \cap \mathbb{A}^{n+1} \backslash$ \{diagonals\} is stable under affine transformations, in particular, rescaling and translation.

Consequently, substituting $x_{i} \mapsto\left(x_{i}+t\right)$ for $1 \leq i \leq n$ to compute the multiplicity along $\operatorname{Diag}_{N}$ leaves $f$ invariant. Since the polynomial is homogeneous, we have that the multiplicity of $f$ along the partial diagonal $\operatorname{Diag}_{N}$ is precisely the degree of $f$, as was to be shown.

We now introduce notation to facilitate comparison of the class of $D \subset \bar{M}_{0, n}$ and that of $\pi_{n+1}^{-1}(D) \subset \bar{M}_{0, n+1}$. Let $d_{I}$ and $\delta_{I}$ denote the boundary divisors on $\bar{M}_{0, n}$ and $\bar{M}_{0, n+1}$, respectively. For $r \in\{1, \ldots, n\}$ and $I \subset\{1, \ldots, n\} \backslash\{r\}$ with $1 \leq$ $|I| \leq n-4$, let $e_{I}^{r}=d_{I \cup\{r\}}$, and let $h \subset \bar{M}_{0, n}$ denote the pull-back of a hyperplane under the Kapranov morphism in index $r$. Let $E_{I}^{n+1}=\delta_{I \cup\{n+1\}}$, and let $H$ be the pull-back of a hyperplane under $\psi_{n+1}: \bar{M}_{0, n+1} \rightarrow \mathbb{P}^{n-2}$.

Proposition 2.4. Let $D \subset \bar{M}_{0, n}$ be an irreducible divisor. Suppose that

$$
\pi_{n+1}^{-1}(D) \sim a H-\sum_{1 \leq|I| \leq n-3} m_{I} E_{I}
$$

on $\bar{M}_{0, n+1}$, where $\pi_{n+1}: \bar{M}_{0, n+1} \rightarrow \bar{M}_{0, n}$ is the forgetful morphism in index $n+1$. Then

$$
D \sim m_{\{r\}} h-\sum_{\substack{2 \leq|I| \leq n-3 \\ r \in I}} m_{I} e_{I \backslash\{r\}}^{r}
$$

as a divisor on $\bar{M}_{0, n}$, with notation as in the paragraph preceding the result.

Proof. For concreteness, assume that $r=1$. The argument centers on computing classes of pull-backs of free generators $e_{I}$ and $h$ under $\pi_{n+1}$. The proposition is a straightforward calculation, which appeals to three basic facts:

(i) $\pi_{n+1}^{-1}\left(d_{J}\right) \sim \delta_{J \cup\{n+1\}}+\delta_{J}$.

(ii) $h \sim \sum_{a, s \in F, 1 \notin F} d_{F}$ for any distinct $a, s$ in $\{2, \ldots, n\}$.

(iii) $\delta_{\{i, j\}} \sim H-\sum_{i, j \notin F, 2 \leq|F| \leq n-3} E_{F}$ for $i<j \in\{1, \ldots, n\}$. 
As previously discussed, (i) follows from noting that $\pi_{n+1}$ has reduced fibers; (ii) is proved in [KT, §3.4]; (iii) is a reformulation of (ii) applied to divisors on $\bar{M}_{0, n+1}$. Now consider $e_{I}=\delta_{I \cup\{1\}}$ for $I \subset\{2, \ldots, n\}$ with $1 \leq|I| \leq n-4$. In the case that $2 \leq|I| \leq n-4$, we have

$$
\pi_{n+1}^{-1}\left(d_{I \cup\{1\}}\right) \sim \delta_{I \cup\{1, n+1\}}+\delta_{I \cup\{1\}} \sim E_{I \cup\{1\}}^{n+1}+E_{\{2, \ldots, n\} \backslash I}^{n+1},
$$

appealing to (i). If $I=\{i\}$, then

$$
\pi_{n+1}^{-1}\left(d_{\{i, 1\}}\right) \sim \delta_{\{i, 1, n+1\}}+\delta_{\{i, 1\}} \sim E_{\{1, i\}}^{n+1}+H-\sum_{\substack{1, i \notin J \\ 2 \leq|J| \leq n-3}} E_{J}^{n+1},
$$

using (iii) for the last equivalence. Last, we compute

$$
\pi_{n+1}^{-1}(h) \sim \sum_{\substack{a, s \in F \\ 1 \notin F}} \pi_{n+1}^{-1}\left(d_{F}\right) .
$$

Applying (i), we obtain

$$
\begin{aligned}
\pi_{n+1}^{-1}(h) \sim \sum_{\substack{a, s \in F \\
1 \notin F \\
2 \leq|F| \leq n-3}} \delta_{F \cup\{n+1\}}+\sum_{\substack{a, s \in F \\
1 \notin F \\
|F|=n-2}} \delta_{F \cup\{n+1\}}+\sum_{\substack{a, s \in F \\
1, n+1 \notin F \\
2 \leq \leq F \mid \leq n-2}} \delta_{F} \\
\sim \sum_{\substack{a, s \in F \\
1 \notin F \\
2 \leq|F| \leq n-3}} \delta_{F \cup\{n+1\}}+\sum_{\substack{a, s \in F \\
1 \notin F \\
|F|=n-2}} \delta_{F \cup\{n+1\}} \\
+\sum_{\substack{a, s \in F \\
n+1 \notin F \\
2 \leq|F| \leq n-1}} \delta_{F}-\sum_{\substack{a, s, 1 \in F \\
n+1 \notin F \\
2 \leq|F| \leq n-1}} \delta_{F}-\delta_{\{2, \ldots, n\}} .
\end{aligned}
$$

Note that the term involving $\delta_{\{2, \ldots, n\}}=\delta_{\{1, n+1\}}=E_{\{1\}}$ in (12) must be subtracted because the last term in (11) includes only $\delta_{F}$ for $|F| \leq n-2$.

Using (ii), (12) can be rewritten as

$$
\begin{aligned}
& \sum_{\substack{a, s \in F \\
|F| \geq 2 \\
1 \notin F}} E_{F}^{n+1}+\sum_{i \notin\{a, s, 1, n+1\}}\left(H-\sum_{\substack{1, i \notin J \\
2 \leq|J| \leq n-3}} E_{J}^{n+1}\right)+H \\
&-\sum_{\substack{2 \leq|F| \leq n-3 \\
a, s, 1 \notin F}} E_{F}^{n+1}-E_{\{1\}}^{n+1} \\
&=-E_{\{1\}}^{n+1}+\Omega,
\end{aligned}
$$

where $\Omega$ absorbs terms proportional to $H$ or $E_{J}$ for $1 \notin J$. Using (10), (9), and (13), we see that

$$
\pi_{n+1}^{-1}\left(b h-\sum_{1 \leq|I| \leq n-4\}} k_{I} e_{I}\right) \sim-b E_{\{1\}}^{n+1}-\sum_{\substack{I \subset\{1, \ldots, n\} \\ 2 \leq|I| \leq n-4}} k_{I} E_{I \cup\{1\}}^{n+1}+\Omega,
$$


where again terms in $\Omega$ are linearly independent of those explicitly written. Hence, we have $b=m_{\{1\}}$ and $k_{I}=m_{I \cup\{1\}}$, as was to be shown.

Corollary 2.5. If $D \subset \bar{M}_{0, n}$ is specified by $f \in k\left[x_{1}, \ldots, x_{n}\right]$ as in Theorem 2.2, the class of $D$ in the index $r$ Kapranov basis for $\mathrm{Cl}\left(\bar{M}_{0, n}\right)$ is

$$
m_{\{r\}} H-\sum_{1 \leq|I| \leq n-3} m_{I \cup\{r\}} E_{I},
$$

where $m_{J}$ is the multiplicity of $V(f)$ along the partial diagonal $\operatorname{Diag}_{\{1, \ldots, n\} \backslash J}$.

The following Macaulay2 code uses the formulae derived before to give the class of a divisor specified by a polynomial equation with respect to the Kapranov basis in index $n+1$. It is important to note that the result of this calculation is in fact the divisor class modulo a large prime. For small $n$ and low-degree polynomials, this is unlikely to result in discrepancies with the actual class. Moreover, the code is best suited for experimentation and motivation; in this context, sufficient certainty about a given class can be obtained by varying the modulus.

To implement the code, first import the code into Macaulay2. Then define a polynomial $f=f\left(x_{1}, \ldots, x_{n}\right)$. The command $T(f)$ outputs the class encoded as a polynomial as follows: the class $H$ is represented by a variable $z$, and the classes $E_{I}$ are represented as monomials $\prod_{i \in I} x_{i}$.

A brief explanation of the code: the first part creates an $n \times 2^{n}$ binary matrix $V$ encoding partial diagonals. The diagonal $\left\{x_{i}=x_{j} \mid i, j \in I\right\}$ corresponds to the row with $1 \mathrm{~s}$ in the column corresponding to indices in $I$ and zeroes elsewhere. The associated matrix $W$ omits partial diagonals along which multiplicities need not be calculated.

Using this matrix $W$, the second part of the code defines functions (taking as input polynomials) that are composed to calculate the multiplicity along relevant diagonals. More explicitly, the code first performs a change of variables and then calculates the degree of the resulting polynomial, viewed as a polynomial in the variable $t$.

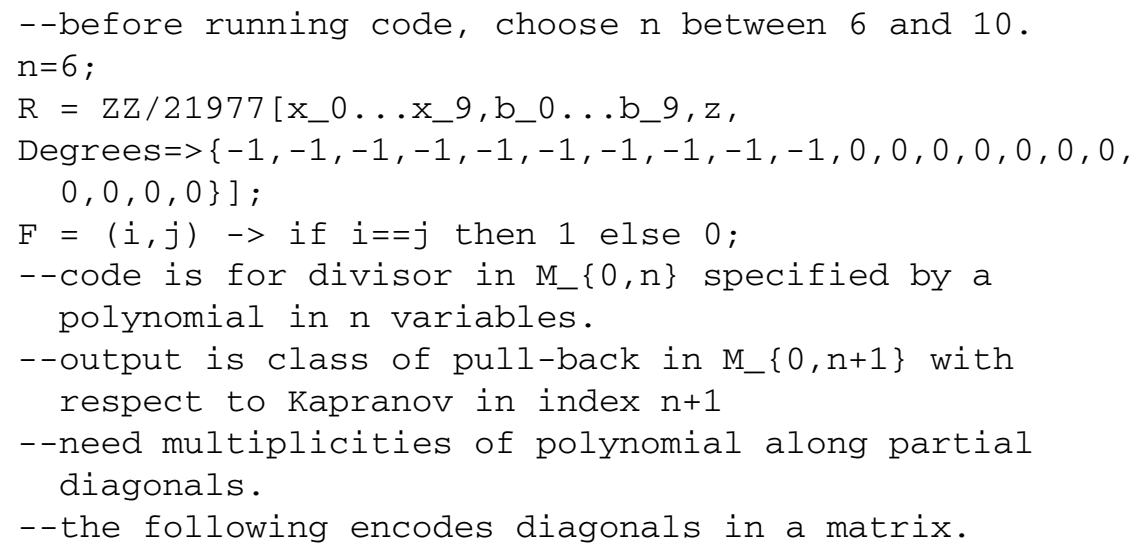




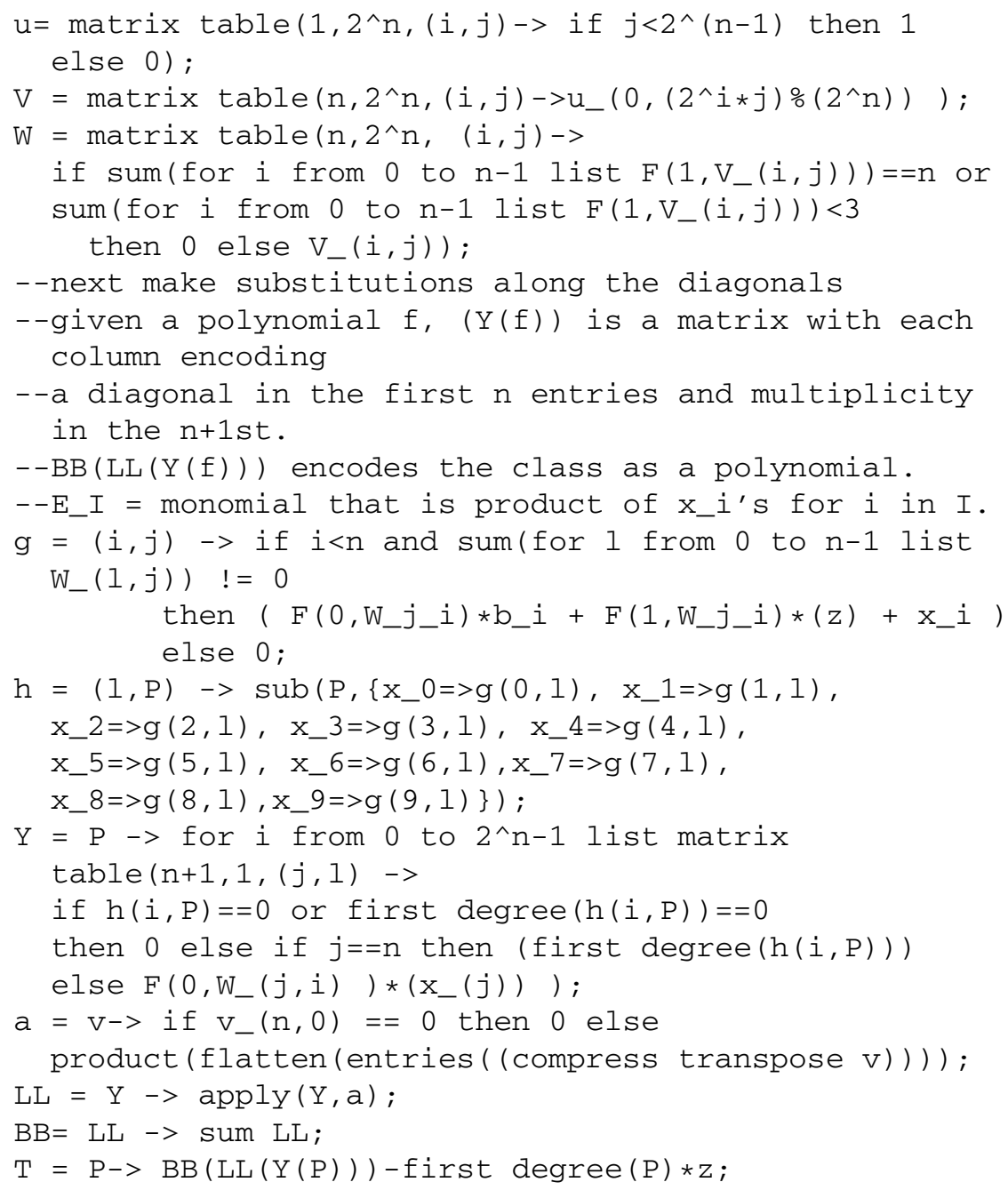

\section{Equations of Hypertree Divisors}

The following definitions are from [CT]. A hypertree on a set $N$ is a collection $\Gamma=\left\{\Gamma_{1}, \ldots, \Gamma_{d}\right\}$ of subsets of $N$ satisfying:

(1) For any $j \in\{1, \ldots, d\},\left|\Gamma_{j}\right| \geq 3$.

(2) Each $i \in N$ is contained in at least two distinct $\Gamma_{j} \mathrm{~s}$.

(3) Convexity: $\left|\bigcup_{j \in S} \Gamma_{j}\right|-2 \geq \sum_{j \in S}\left(\left|\Gamma_{j}\right|-2\right)$ for any $S \subset\{1, \ldots, d\}$.

(4) Normalization: $|N|-2=\sum_{1 \leq j \leq d}\left(\left|\Gamma_{j}\right|-2\right)$.

A hypertree $\Gamma$ is irreducible if the convexity condition (3) is strict for $1<|S|<d$. A planar realization of a hypertree $\Gamma=\left\{\Gamma_{1}, \ldots, \Gamma_{d}\right\}$ is a collection of points $p_{1}, \ldots, p_{n} \in \mathbb{P}^{2}$ such that $p_{i}, p_{j}, p_{k}$ are collinear if and only if there exists $\alpha \in\{1, \ldots, d\}$ such that $i, j, k \in \Gamma_{\alpha}$. 


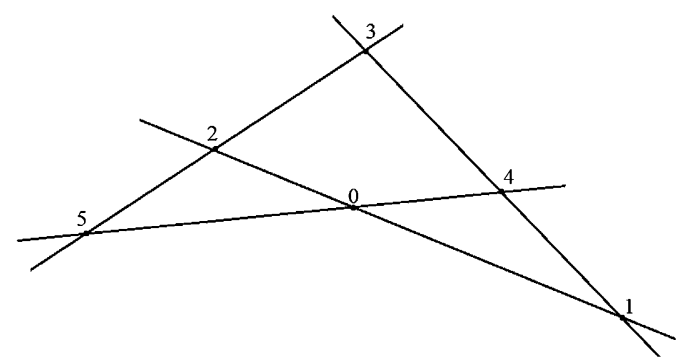

Figure 2 Planar realization of the complete quadrilateral, defined by $\Gamma=\{012,314,045,325\}$

Given planar realization, the images of $p_{1}, \ldots, p_{n}$ under projection from a general point give $n$ distinct marked points on $\mathbb{P}^{1}$. Given $\Gamma$, define the hypertree divisor $D_{\Gamma} \subset \bar{M}_{0, n}$ as the closure of the locus

$\left\{\left[\mathbb{P}^{1} ; q_{1}, \ldots, q_{n}\right] \mid \exists\right.$ a realization $\left\{p_{i}\right\}$ and projection $\pi$ with $\left.q_{i}=\pi\left(p_{i}\right)\right\}$.

For $\Gamma$ irreducible, Castravet and Tevelev show that $D_{\Gamma}$ is a nonempty irreducible divisor generating an extremal ray of $\overline{\operatorname{Eff}}\left(\bar{M}_{0, n}\right)$.

Rather than defining $D_{\Gamma}$ as before, we might consider the closure of the locus of equivalence classes $\left[\mathbb{P}^{1} ; q_{1}, \ldots, q_{n}\right]$ such that $q_{i}$ s are projections of points $\left\{p_{1}, \ldots, p_{n}\right\} \subset \mathbb{P}^{2}$ where $p_{i}, p_{j}, p_{k}$ are collinear if $i, j, k \subset \Gamma_{\alpha}$ and not all $p_{i}$ are collinear. The distinction here is that we no longer require "only if". It is nontrivial that this weaker definition coincides with that of $D_{\Gamma}$ and is proved in [CT, §4]. We will use this characterization to obtain equations in $k\left[x_{1}, \ldots, x_{n}\right]$ specifying irreducible hypertree divisors (where this specification in the precise sense is discussed in Section 2). Our proof is a direct generalization of results in [CT] for the case where all subsets comprising the hypertree have three elements.

We first set up some notation. Given a subset $\Gamma_{i}=\left\{a_{i 1}, \ldots, a_{i k_{i}}\right\}$, let $\Gamma_{i j}=$ $\left\{a_{i 1}, a_{i 2}, a_{i j}\right\}$ for $3 \leq j \leq k_{i}-2$. By normalization

$$
\sum_{i=1}^{d}\left|\Gamma_{i}\right|=\sum_{i=1}^{d}\left(k_{i}-2\right)=n-2
$$

and from each $\Gamma_{i}$ we define precisely $k_{i}-2$ sets $\Gamma_{i j}$, so the total number of subsets $\Gamma_{i j}$ for $1 \leq i \leq d, 3 \leq j \leq k_{i}$ is $n-2$. Let $\left\{G_{\alpha}\right\}_{1 \leq \alpha \leq n-2}$ to be an ordering of the collection of $\Gamma_{i j}$ s. With this, we can state the following:

THEOREM 3.1. Let $\Gamma=\left\{\Gamma_{1}, \ldots, \Gamma_{d}\right\}$ be a hypertree. With notation as preceding the theorem, define the $(n-2) \times n$ matrix $\mathbf{A}$ by

$$
\begin{aligned}
G_{\alpha} & =\{i, j, k\} \\
& \Longrightarrow \mathbf{A}_{\alpha, i}=\left(x_{j}-x_{k}\right), \quad \mathbf{A}_{\alpha, j}=\left(x_{k}-x_{i}\right), \quad \mathbf{A}_{\alpha, k}=\left(x_{i}-x_{j}\right) .
\end{aligned}
$$

If $\beta \notin G_{\alpha}$, then let $\mathbf{A}_{\alpha . \beta}=0$. Define $\mathbf{B}$ as the $(n-3) \times(n-3)$ matrix obtained from $\mathbf{A}$ by deleting a row and all columns in which the entries of that row are 
nonzero. The hypertree divisor $D_{\Gamma}$ is specified by

$$
\frac{\operatorname{det} \mathbf{B}}{\prod_{i=1}^{d}\left(x_{a_{i 1}}-x_{a_{i 2}}\right)^{k_{i}-3}} .
$$

Proof. The condition that points $x_{1}, \ldots, x_{n} \in \mathbb{A}^{1}$ can be obtained from the projection of a hypertree curve is equivalent to the existence of $y_{1}, \ldots, y_{n} \in \mathbb{A}^{n}$ so that, defining $p_{i}=\left(x_{i}, y_{i}\right)$, the following is satisfied:

Not all $p_{k}$ are collinear, and $i, j, w \in \Gamma_{k} \Longrightarrow p_{i}, p_{j}, p_{w}$ collinear.

By construction, $p_{i}, p_{j}, p_{w}$ are collinear whenever $i, j, w \in \Gamma_{k}$ for some $k$ if and only if $p_{x}, p_{y}, p_{z}$ are collinear whenever $x, y, z \in G_{i}$ for some $i$. We apply the argument given in [CT, §8] to the subsets $G_{i}$ to obtain $\mathbf{A}$ as defined before so that a solution to $\mathbf{A}\left(y_{1}, \ldots, y_{n}\right)^{T}=0$ with not all points $p_{i}$ collinear implies that $\left[\mathbb{P}^{1} ; a_{1}, \ldots, a_{n}\right] \in D_{\Gamma}$ if $\left[\mathbb{P}^{1}: a_{1}, \ldots, a_{n}\right]=\chi\left(x_{1}, \ldots, x_{n}\right)$, where $\chi \mathbb{A}^{1}[n] \longrightarrow \bar{M}_{0, n}$ is as defined in Section 1 .

If a solution $y=\left(y_{1}, \ldots, y_{n}\right)^{T}$ to $\mathbf{A} y=0$ exists, then we may choose coordinates so that three points corresponding to the indices in some fixed $G_{i_{0}}$ lie along $y=0$. We shall subsequently refer to this $G_{i_{0}}$ as a pivot subset. Requiring that not all $p_{i}$ are collinear and setting $y_{i}=0$ for $i \in G_{i_{0}}$, we seek a nontrivial solution $\mathbf{B} y=0$, where $\mathbf{B}$ is as defined in the theorem.

For points $x_{1}, \ldots, x_{n}$, there exists a configuration of points $p_{1}, \ldots, p_{n}$ satisfying (14) if and only if $\operatorname{det} \mathbf{B}\left(x_{1}, \ldots, x_{n}\right)=0$. Let $A$ denote $\mathbb{A}^{n}$ minus the partial diagonals of codimension greater than 1 ; what we have shown is that $\phi_{*}^{-1}(V(\operatorname{det} \mathbf{B}) \cap A)=\chi^{-1}\left(D_{\Gamma} \cap M_{0, n}\right)$ with maps $\phi$ and $\chi$ as defined in Section 2. Hence, $\operatorname{det} \mathbf{B}$ is the correct equation for $D_{\Gamma}$ on $M_{0, n}$, but $\operatorname{det} \mathbf{B}$ may include erroneous boundary factors corresponding to partial diagonals.

Claim 3.2. For each $\Gamma_{i}=\left\{a_{i, 1}, a_{i, 2}, \ldots, a_{i, k_{\alpha}}\right\}, \max \left\{m:\left(x_{a_{i, 1}}-x_{a_{i, 2}}\right)^{m} \mid\right.$ $\operatorname{det} \mathbf{B}\}=k_{\alpha}-3$.

Given the claim, $D_{\Gamma}$ is specified by

$$
g\left(x_{1}, \ldots, x_{n}\right):=\frac{\operatorname{det} \mathbf{B}}{\prod_{i=1}^{d}\left(x_{a_{i, 1}}-x_{a_{i, 2}}\right)^{k_{\alpha}-3}} .
$$

To see that $\phi_{*}^{-1}(V(g))=\chi^{-1}\left(D_{\Gamma}\right)$, note that $\operatorname{deg}(g)=n-2-\sum_{i=1}^{d}\left(k_{i}-2\right)=$ $d-1$, where the last equality invokes normalization of $\Gamma$. Вy [CT, §4.2] we know that $\pi_{n+1}^{-1}\left(D_{\Gamma}\right) \sim(d-1) H+\cdots$. By Theorem 2.2 a divisor $D$ specified by a polynomial $F$ satisfies $\pi_{n+1}^{-1}(D) \sim a H+\cdots$ where $a=\operatorname{deg}(F)$. Hence, degree considerations show $\phi_{*}^{-1}(V(g))=\chi^{-1}\left(D_{\Gamma}\right)$.

We now prove the claim. Consider the rows of $\mathbf{B}$ corresponding to a given subset $\Gamma_{i}=\left\{a_{i 1}, \ldots, a_{i k_{i}}\right\}$. Assume for simplicity that $i=1$ and $a_{i 1}, a_{i 2}, \ldots, a_{i k_{i}}=$ 
$1,2, \ldots, k_{1}$; the argument generalizes. The first $k_{1}-2$ rows of $\mathbf{A}$ are as follows:

$$
\left(\begin{array}{cccccccc}
x_{2}-x_{3} & x_{3}-x_{1} & x_{1}-x_{2} & 0 & 0 & \cdots & 0 & \cdots \\
x_{2}-x_{4} & x_{4}-x_{1} & 0 & x_{1}-x_{2} & 0 & \cdots & 0 & \cdots \\
x_{2}-x_{5} & x_{5}-x_{1} & 0 & 0 & x_{1}-x_{2} & \cdots & 0 & \cdots \\
\vdots & \vdots & \vdots & \vdots & \vdots & \ddots & \vdots & \ddots \\
x_{2}-x_{k_{i}} & x_{k_{i}}-x_{1} & 0 & 0 & 0 & \cdots & x_{1}-x_{2} & \ldots
\end{array}\right) .
$$

In passing from the matrix $\mathbf{A}$ to $\mathbf{B}$, the rows of $\mathbf{A}$ shown can be altered in three ways. Let $G_{i_{0}}$ be the pivot subset used to obtain $\mathbf{B}$.

(1) $G_{i_{0}} \subset \Gamma_{1}$. Without loss of generality, we may assume that $G_{i_{0}}$ corresponds to the first row of $\mathbf{A}$. The first $k_{1}-3$ rows of $\mathbf{B}$ then appear as follows:

$$
\left(\begin{array}{ccccc}
x_{1}-x_{2} & 0 & \cdots & 0 & \cdots \\
0 & x_{1}-x_{2} & \cdots & 0 & \cdots \\
\vdots & \vdots & \vdots & \vdots & \ddots \\
0 & 0 & \cdots & x_{1}-x_{2} & \cdots
\end{array}\right)
$$

Evidently, $\left(x_{1}-x_{2}\right)^{k_{1}-3}$ divides $g$.

(2) $\left|G_{i_{0}} \cap \Gamma_{1}\right|=0$. In this case, the first $k_{1}-2$ rows of $\mathbf{B}$ will be identical to those of A. Adding column 2 to column 1 gives

$$
\left(\begin{array}{cccccccc}
x_{2}-x_{1} & x_{3}-x_{1} & x_{1}-x_{2} & 0 & 0 & \ldots & 0 & \ldots \\
x_{2}-x_{1} & x_{4}-x_{1} & 0 & x_{1}-x_{2} & 0 & \ldots & 0 & \ldots \\
x_{2}-x_{1} & x_{5}-x_{1} & 0 & 0 & x_{1}-x_{2} & \ldots & 0 & \ldots \\
\vdots & \vdots & \vdots & \vdots & \vdots & \ddots & \vdots & \ddots \\
x_{2}-x_{1} & x_{k}-x_{1} & 0 & 0 & 0 & \ldots & x_{1}-x_{2} & \ldots
\end{array}\right)
$$

Expansion across rows shows that $\left(x_{1}-x_{2}\right)^{k_{1}-3}$ divides $\operatorname{det}(\mathbf{B})$.

(3) $\left|G_{i_{0}} \cap \Gamma_{1}\right|=1$. This is the situation where precisely one column and no rows of the submatrix of $\mathbf{A}$ corresponding to $\Gamma_{1}$ are removed in passing to $\mathbf{B}$. Let $\{h\}=G_{i_{0}} \cap \Gamma_{1}$. We have two subcases to consider:

- $3 \leq h$. This results in a submatrix of the first $k_{1}-2$ rows of $\mathbf{B}$ of the form

$$
\left(\begin{array}{ccccccc}
x_{2}-x_{3} & x_{3}-x_{1} & 0 & 0 & \cdots & 0 & \cdots \\
x_{2}-x_{4} & x_{4}-x_{1} & x_{1}-x_{2} & 0 & \cdots & 0 & \cdots \\
x_{2}-x_{5} & x_{5}-x_{1} & 0 & x_{1}-x_{2} & \cdots & 0 & \cdots \\
\vdots & \vdots & \vdots & \vdots & \ddots & \vdots & \ddots \\
x_{2}-x_{k_{i}} & x_{k_{i}}-x_{1} & 0 & 0 & \cdots & x_{1}-x_{2} & \cdots
\end{array}\right) .
$$

The argument from case 2 goes through (with minor adjustments) to show that $\left(x_{1}-x_{2}\right)^{k_{1}-3}$ is a factor of $\operatorname{det} \mathbf{B}$.

- $h=1$ or $h=2$. 
This results in the first $k_{1}-2$ rows of $\mathbf{B}$ of the form

$$
\left(\begin{array}{ccccccc}
x_{3}-x_{1} & x_{1}-x_{2} & 0 & 0 & \ldots & 0 & \cdots \\
x_{4}-x_{1} & 0 & x_{1}-x_{2} & 0 & \ldots & 0 & \ldots \\
x_{5}-x_{1} & 0 & 0 & x_{1}-x_{2} & \ldots & 0 & \ldots \\
\vdots & \vdots & \vdots & \vdots & \ddots & \vdots & \ddots \\
x_{k_{i}}-x_{1} & 0 & 0 & 0 & \ldots & x_{1}-x_{2} & \ldots
\end{array}\right) .
$$

Evidently, $\left(x_{1}-x_{2}\right)^{k_{1}-3}$ divides $\operatorname{det} \mathbf{B}$.

This proves the claim.

All hypertrees up to permutation for at most 11 vertices were found in [Sch]. Enumeration of small irreducible hypertrees is as follows: 1 for 6 or 7 vertices; 3 for 8 vertices; 11 for 9 vertices; and 96 for 10 vertices.

Using our Macaulay2 program for computing classes specified by polynomial equations (see Section 2) and the polynomial (15), we computed all divisor classes corresponding to irreducible hypertrees for $6 \leq n \leq 10$. We additionally wrote a program to compute symmetry group sizes and computed symmetry groups of irreducible hypertree classes for $6 \leq n \leq 8$.

Particularly nice hypertrees are obtained via even triangulations of a twosphere: given a bicolored (say black and white) triangulation of the two-sphere with $n$ vertices, we can consider unordered triplets $\{i, j, k\}$ corresponding to the vertices of black triangles. The collection of all such triplets gives a set of subsets of $\{1, \ldots, n\}$; Castravet and Tevelev show that, for any bicolored triangulation, this collection of subsets yields a hypertree. They call hypertrees obtained in this way spherical hypertrees. These spherical hypertrees are irreducible unless the triangulation is a connected sum [CT, 1.6]. For $6 \leq n \leq 10$, we classify spherical hypertrees in our database. Spherical hypertrees are further discussed in Theorem 4.9: certain spherical hypertree divisors are seen to arise as certain Chen-Coskun divisors.

For the complete database and Macaulay2 code specific to hypertree divisors, see [Op]. We hope that these data will prove useful in further investigations of hypertrees and other divisors. In addition to the production of the database, the code from the previous section was applied to explore properties of divisors, motivating the discovery of our counterexamples to Conjecture 1.1. We provide the counterexample in Section 5 but must first describe the family of divisors used in the construction.

\section{Chen-Coskun Divisors}

Chen and Coskun [CC] define divisors on the moduli space $\bar{M}_{1, n}$ of genus 1 curves with $n$ ordered markings as follows. Given an $n$-tuple of integers

$$
\mathbf{a}=\left(a_{1}, \ldots, a_{n}\right)
$$

with $\sum_{i} a_{i}=0$, define $D_{\mathbf{a}} \subset \bar{M}_{1, n}$ to be the closure of the locus of smooth genus 1 curves $\left[E ; p_{1}, \ldots, p_{n}\right]$ so that $\sum a_{i} p_{i}=0$ in the Jacobian of the curve. Results 

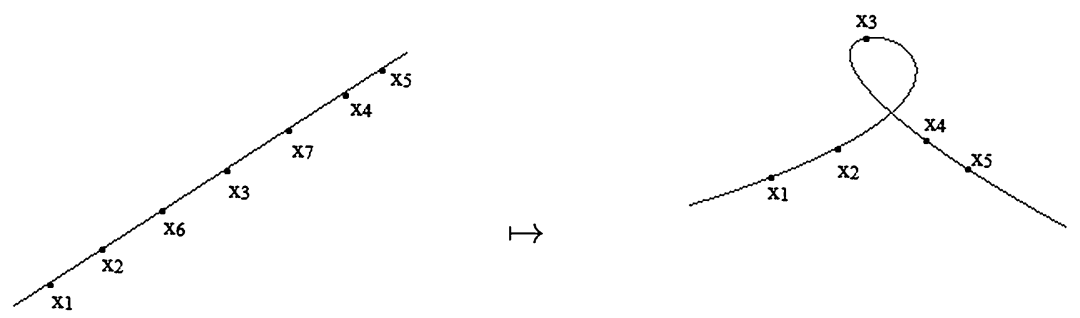

Figure 3 The clutching morphism $\varphi: \bar{M}_{0,7} \rightarrow \bar{M}_{1,5}$

on these divisors include that, for $\operatorname{gcd}\left(a_{1}, \ldots, a_{n}\right)=1$ and $n \geq 3$, the divisor $D_{\mathbf{a}}$ is an irreducible, rigid effective divisor generating an extremal ray of the effective cone of $\bar{M}_{1, n}$. Moreover, there are infinitely many of distinct divisors of this form on $\bar{M}_{1, n}$ for each $n \geq 4$, showing that $\overline{\operatorname{Eff}}\left(\bar{M}_{1, n}\right)$ is not finitely generated [CC].

The natural clutching morphism $\varphi: \bar{M}_{0, n+2} \longrightarrow \bar{M}_{1, n}$ identifies marked points $p_{n+1}$ and $p_{n+2}$ on a rational curve in $\bar{M}_{0, n+2}$ (see Figure 3 ). We might ask what can be said about the proper transforms under $\varphi$ of the divisors defined in [CC]. However, the definition given by Chen and Coskun does not lend itself to study of these proper transforms: the image $\varphi\left(\bar{M}_{0, n+2}\right)$ lies entirely in the complement of the smooth locus, and their definition is in terms of the closure of a collection of smooth curves. Hence, we give an alternate definition entirely within the locus of nodal genus 1 curves.

For $\mathbf{a}=\left(a_{1}, \ldots, a_{n}\right) \in \mathbb{Z}^{n}$ satisfying $\sum_{i=1}^{n} a_{i}=0$ and $\operatorname{gcd}\left(a_{1}, \ldots, a_{n}\right)=1$, define $D_{\mathbf{a}}$ as the closure in $\varphi\left(\bar{M}_{0, n+2}\right)$ of the locus of irreducible nodal curves $\left[C ; p_{1}, \ldots, p_{n}\right]$ with $n$ distinct smooth markings such that $\sum_{i=1}^{n} a_{i} p_{i}=0$ in $\operatorname{Pic}^{0}(C) \simeq \mathbb{G}_{m}$. It is clear that our subset $D_{\mathbf{a}} \subset \varphi\left(\bar{M}_{0, n+2}\right)$ is the intersection of the divisor $D_{\mathbf{a}}$ defined in [CC] with $\varphi\left(\bar{M}_{0, n+2}\right)$, but we will not use this fact. Henceforth, $D_{\mathbf{a}}$ will refer to our divisor defined on $\varphi\left(\bar{M}_{0, n+2}\right)$ unless otherwise noted.

Lemma 4.1. The locus $D_{\mathbf{a}} \subset \varphi\left(\bar{M}_{0, n+2}\right)$ is an irreducible divisor.

Proof. Let $Y=\mathbb{G}_{m}^{n} \backslash\{$ diagonals . Consider the following commutative diagram:

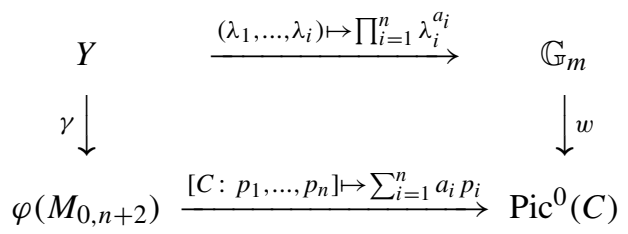

Here $\gamma$ is induced by an isomorphism of the smooth locus of an irreducible nodal cubic with $\mathbb{G}_{m}$ and maps an $n$-tuple of distinct points to their isomorphism class in $\varphi\left(\bar{M}_{0, n+2}\right) \subset \bar{M}_{1, n}$. The map $w$ is the canonical identification of $\operatorname{Pic}^{0}(C)$ with 
$\mathbb{G}_{m}$. Note that $\gamma$ is surjective onto $\varphi\left(M_{0, n+2}\right)$. Define

$$
S=\left\{\left(p_{1}, \ldots, p_{n}\right) \in Y \mid \prod_{i=1}^{n} p_{i}^{a_{i}}=1\right\}
$$

and $\gamma^{-1}\left(D_{\mathbf{a}} \cap \varphi\left(M_{0, n+2}\right)\right)=S$. Hence, it suffices to show irreducibility of $S$.

Recall that endomorphisms of $\mathbb{G}_{m}^{n}$ are given by

$$
p_{i} \mapsto \prod_{j=1}^{n} p_{j}^{r_{i j}}
$$

for $r_{i j} \in \mathbb{Z}$; so we represent an endomorphism via an integral matrix acting on exponents:

$$
R=\left(\begin{array}{cccc}
r_{11} & r_{12} & \cdots & r_{1 n} \\
r_{21} & r_{22} & \cdots & r_{2 n} \\
r_{31} & r_{32} & \cdots & r_{3 n} \\
\vdots & \vdots & \vdots & \vdots \\
r_{n 1} & r_{n 2} & \cdots & r_{n n}
\end{array}\right)
$$

The corresponding map is an automorphism if and only if $|\operatorname{det} R|=1$.

Suppose that there is an automorphism $h$ such that

$$
p_{1} \stackrel{h}{\mapsto} \prod_{i=1}^{n} p_{i}^{a_{i}}
$$

Then

$$
\left\{\left(p_{1}, \ldots, p_{n}\right) \mid p_{1}^{a_{1}} \cdots p_{n}^{a_{n}}=1\right\} \simeq\left\{\left(q_{1}, \ldots, q_{n}\right) \mid q_{1}=1\right\},
$$

where the isomorphism is induced by the given endomorphism. This is the graph of a morphism from $\mathbb{G}_{m}^{n-1}$ to $\mathbb{G}_{m}$ and hence an irreducible divisor. So it suffices to show that there exists an automorphism with matrix $R$ such that $r_{i 1}=a_{i}$ for $1 \leq$ $i \leq n$. We show this by induction on $n$. For $n=2$, the condition that $\operatorname{gcd}\left(a_{1}, a_{2}\right)=$ 1 gives that there exist $c_{1}, c_{2}$ such that $a_{1} c_{1}-a_{2} c_{2}=1$. A matrix with the desired property is then given by

$$
R=\left(\begin{array}{ll}
a_{1} & c_{2} \\
a_{2} & c_{1}
\end{array}\right) .
$$

Now consider the case for $S \subset \mathbb{G}_{m}^{k+1}$ with $\operatorname{gcd}\left(a_{1}, \ldots, a_{k+1}\right)=1$. Let $s:=$ $\operatorname{gcd}\left(a_{1}, \ldots, a_{k}\right)$. Factoring out the $\mathrm{gcd}$, inductively there is an automorphism of $\theta$ of $\mathbb{G}_{m}^{k}$ taking $p_{1}^{a_{1}} \cdots p_{k}^{a_{k}} \stackrel{\theta}{\mapsto} q_{1}^{s}$, where $q_{i}:=\theta\left(p_{i}\right)$. The map $\theta$ extends to an automorphism of $\mathbb{G}_{m}^{k+1}$ with $p_{k+1} \stackrel{\theta}{\mapsto} p_{k+1}$, and we have

$$
S \simeq\left\{\left(q_{1}, \ldots, q_{k+1}\right) \mid q_{1}^{s} q_{k+1}^{a_{k+1}}=1\right\} .
$$

The assumption that $\operatorname{gcd}\left(a_{1}, \ldots, a_{k+1}\right)=1$ forces $\operatorname{gcd}\left(s, a_{k+1}\right)=1$. Hence, the induction is completed by applying the $k=2$ case to obtain an appropriate automorphism of $\left\langle q_{1}, q_{k+1}\right\rangle \simeq \mathbb{G}_{m}^{2}$.

We now give explicit formulas for the class of the proper transform of $D_{\mathbf{a}}$ with respect to the clutching morphism $\varphi$. 
THEOREM 4.2. Given $\mathbf{a}=\left(a_{1}, \ldots, a_{n}\right)$ with $\sum_{i} a_{i}=0$, the proper transform $\Lambda_{\mathbf{a}}$ of $D_{\mathbf{a}}$ under the map $\varphi: \bar{M}_{0, n+2} \rightarrow \bar{M}_{1, n}$ identifying marked points $n+1$ and $n+2$ is an is an irreducible divisor. Furthermore, $\Lambda_{\mathbf{a}}$ is specified in the sense of Section 2 by the polynomial

$$
\begin{gathered}
\frac{1}{x_{n+1}-x_{n+2}} \cdot\left(\prod_{a_{i} \geq 0}\left(x_{n+1}-x_{i}\right)^{\left|a_{i}\right|} \prod_{a_{i} \leq 0}\left(x_{n+2}-x_{i}\right)^{\left|a_{i}\right|}\right. \\
\left.-\prod_{a_{i} \leq 0}\left(x_{n+1}-x_{i}\right)^{\left|a_{i}\right|} \prod_{a_{i} \geq 0}\left(x_{n+2}-x_{i}\right)^{\left|a_{i}\right|}\right),
\end{gathered}
$$

and

with coefficients as follows:

$$
\pi_{n+3}^{-1}\left(\Lambda_{\mathbf{a}}\right) \sim d H-\sum m_{I} E_{I}^{n+3},
$$

- If $n+1, n+2 \notin I$ and $\left\{i \mid a_{i} \neq 0\right\} \subset I$, then $m_{I}=0$.

- If $n+1, n+2 \notin I$ and $\left\{i \mid a_{i} \neq 0\right\} \not \subset I$, then $m_{I}=\left(\sum_{i \notin I}\left|a_{i}\right|\right)-1$.

- If $n+1, n+2 \in I$ and $\left\{i \mid a_{i} \neq 0\right\} \subset\{1, \ldots, n\} \backslash I$, then $m_{I}=1$.

- If $n+1, n+2 \in I$ and $\left\{i \mid a_{i} \neq 0\right\} \not \subset\{1, \ldots, n\} \backslash I$, then $m_{I}=0$.

- If $|\{n+1, n+2\} \cap I|=1$, then $m_{I}=\min \left\{\sum_{0 \leq a_{i} \notin I}\left|a_{i}\right|, \sum_{0 \geq a_{i} \notin I}\left|a_{i}\right|\right\}$.

- $d=\left(\sum_{i}\left|a_{i}\right|\right)-1$.

This theorem immediately yields a number of useful formulae, which we record prior to proving the theorem.

COROLlary 4.3. If $\mathbf{a}=\left(a_{1}, \ldots, a_{n}\right)$ with $a_{i} \neq 0$ for all $i$, then $\pi_{n+3}^{-1}\left(\Lambda_{\mathbf{a}}\right) \sim d H-$ $\sum_{i} m_{I} E_{I}^{n+3}$, with coefficients as follows:

- If $n+1, n+2 \notin I$, then $m_{I}=\left(\sum_{i \notin I}\left|a_{i}\right|\right)-1$.

- If $n+1, n+2 \in I$, then $m_{I}=0$ except when $I=\{n+1, n+2\}$, in which case $m_{I}=1$.

- If $|\{n+1, n+2\} \cap I|=1$, then $m_{I}=\min \left\{\sum_{0 \leq a_{i} \notin I}\left|a_{i}\right|, \sum_{0 \geq a_{i} \notin I}\left|a_{i}\right|\right\}$.

- $d=\left(\sum_{i}\left|a_{i}\right|\right)-1$.

Proof. A special case of Theorem 4.2.

Corollary 4.4. Given $\mathbf{a}=\left(a_{1}, \ldots, a_{n}\right)$ with $\sum_{i} a_{i}=0$, the class of $\Lambda_{\mathbf{a}}$ with respect to the Kapranov basis in index $r$ is

$$
\begin{aligned}
& \left(\sum_{\substack{1 \leq i \leq n \\
i \neq r}}\left|a_{i}\right|-1\right) H-\sum_{\substack{n+1, n+2 \notin I \\
\left\{i \mid a_{i} \neq 0\right\} \not \subset I}}\left(\sum_{i \notin I \cup\{r\}}\left|a_{i}\right|-1\right) E_{I} \\
& -\sum_{\substack{|\{n+1, n+2\} \cap I|=1\\
}} \min \left\{\sum_{\substack{0 \leq a_{i} \\
i \notin I \cup\{r\}}}\left|a_{i}\right|, \sum_{\substack{0 \leq a_{i} \\
i \notin I \cup\{r\}}}\left|a_{i}\right|\right\} E_{I} \\
& -\sum_{\substack{n+1, n+2 \in I \\
I \cap N \subset\left\{i \mid a_{i}=0\right\} \\
r \notin I}} E_{I} .
\end{aligned}
$$


Proof. Apply Proposition 2.4 to Theorem 4.2. Note that the last terms in (17), that is, those involving $E_{I}$ for $\{n+1, n+2\} \in I$, vanish if $a_{r} \neq 0$.

Corollary 4.5. Suppose that $\mathbf{a}=\left(a_{1}, \ldots, a_{n}\right)$ with $a_{i} \neq 0$ for all $i$. Then the class of $\Lambda_{\left(a_{1}, \ldots, a_{n}\right)} \subset \bar{M}_{0, n+2}$ with respect to the Kapranov basis in index $r$ for $r \in\{1, \ldots, n\}$ is

$$
\begin{aligned}
& \left(\sum_{\substack{1 \leq i \leq n \\
i \neq r}}\left|a_{i}\right|-1\right) H-\sum_{n+1, n+2 \notin I}\left(\sum_{i \notin I \cup\{r\}}\left|a_{i}\right|-1\right) E_{I} \\
& -\sum_{|\{n+1, n+2\} \cap I|=1} \min \left\{\sum_{\substack{0 \leq a_{i} \\
i \notin I \cup\{r\}}}\left|a_{i}\right|, \sum_{\substack{0 \geq a_{i} \\
i \notin I \cup\{r\}}}\left|a_{i}\right|\right\} E_{I} .
\end{aligned}
$$

Proof. Apply Proposition 2.4 to Theorem 4.3.

We now prove the main result.

Proof of Theorem 4.2. We can describe the interior of $\Lambda_{\mathbf{a}}$ as follows:

$$
\begin{aligned}
& {\left[\mathbb{P}^{1} ; p_{1}, \ldots, p_{n+2}\right] \in \Lambda_{\mathbf{a}} \cap M_{0, n+2} } \\
& \Longleftrightarrow \varphi\left(\left[\mathbb{P}^{1} ; p_{1}, \ldots, p_{n+2}\right]\right)=\left[C ; q_{1}, \ldots, q_{n}\right] \in D_{\mathbf{a}} \\
& \Longleftrightarrow \exists g \in k(C): \operatorname{div}(g)=\sum_{i=1}^{n} a_{i} q_{i},
\end{aligned}
$$

for $g$ regular and invertible at the node

$$
\Longleftrightarrow \quad \exists h \in k\left(\mathbb{P}^{1}\right): \operatorname{div}(h)=\sum_{i=1}^{n} a_{i} p_{i} \quad \text { and } \quad h\left(p_{n+1}\right)=h\left(p_{n+2}\right) \text {. }
$$

Evidently, $h(x)=\prod_{i=1}^{n}\left(x-p_{i}\right)^{a_{i}}$ on an appropriate affine chart for a representative of $\left[\mathbb{P}^{1} ; p_{1}, \ldots, p_{n+2}\right]$. Thus, the condition that

$$
x=\left[\mathbb{P} ; p_{1}, \ldots, p_{n+2}\right] \in \Lambda_{\mathbf{a}}
$$

is equivalent to requiring that any $\left(\mathbb{A}^{1} ; q_{1}, \ldots, q_{n+2}\right)$ mapping to $x$ under the map $\chi$ from the Fulton-MacPherson configuration space satisfies $h\left(q_{n+1}\right)=h\left(q_{n+2}\right)$. This gives an equation $F$ specifying $\Lambda_{\mathbf{a}} \cap M_{0, n+2}$ :

$$
\begin{aligned}
\prod_{a_{i} \geq 0}\left(x_{n+1}-x_{i}\right)^{\left|a_{i}\right|} \prod_{a_{i} \leq 0}\left(x_{n+2}-x_{i}\right)^{\left|a_{i}\right|} \\
\\
\quad-\prod_{a_{i} \leq 0}\left(x_{n+1}-x_{i}\right)^{\left|a_{i}\right|} \prod_{a_{i} \geq 0}\left(x_{n+2}-x_{i}\right)^{\left|a_{i}\right|} .
\end{aligned}
$$

Note that Lemma 4.1 implies that $\Lambda_{\mathbf{a}}=\varphi_{*}^{-1}\left(D_{\mathbf{a}}\right)$ is irreducible. Since $F$ is the correct equation for $\Lambda_{\mathbf{a}}$ on $M_{0, n+2}$, only boundary terms of the form $x_{i}-x_{j}$ for $i \neq j$ can divide $F$. 
Claim 4.6. For $m \in \mathbb{N},\left(x_{i}-x_{j}\right)^{m}$ divides $F$ if and only if

$$
\{i, j\}=\{n+1, n+2\}
$$

and $m=1$.

We obtain Claim 4.6 in the course of proving the formula for classes: the claim is equivalent to the assertion that the multiplicity of $F$ along $\operatorname{Diag}_{\{i, j\}}$ is zero unless $\{i, j\}=\{n+1, n+2\}$, in which case it is 1 . Given the claim, we recover the equation of the theorem.

With notation as from Theorem 2.2, recall that given $f \in k\left[x_{1}, \ldots, x_{n+2}\right]$ such that $\phi_{*}^{-1}(V(f))=\chi^{-1}(D)$, the class of the pull-back $\pi_{n+3}^{-1}(D) \subset \bar{M}_{0, n+3}$ is

$$
d H-\sum_{\substack{1 \leq|I| \leq n-1 \\ I \subset\{1, \ldots, n+2\}}} m_{I} E_{I},
$$

where $d$ is the degree of $f$, and $m_{I}$ is the multiplicity of $f$ along the partial $\operatorname{diagonal}_{\operatorname{Diag}}=\left\{x_{i}=x_{j} \mid i, j \in J\right\}$ for $J=\{1, \ldots, n+3\} \backslash I$. Hence, we must compute the multiplicity of $F$ from (20) along partial diagonals Diag ${ }_{J}$ with $4 \leq$ $|J| \leq n+2$. The multiplicity along a diagonal will be the multiplicity at a general point. To compute the multiplicity at an arbitrary point $b=\left(-b_{1}, \ldots,-b_{n+2}\right)$, we make the substitution $x_{i} \mapsto x_{i}+b_{i}$ and determine the degree of the initial term of the resulting equation as a polynomial in $x_{i}$. To get the multiplicity at a general point $b \in \operatorname{Diag}_{J}$, we set $b_{i}=t$ for $i \in J$ and then compute the minimum degree among nonzero monomials as a polynomial in $x_{i}$.

There are several cases to consider. Throughout, we define $k_{J}$ to be the multiplicity of $F$ along a partial diagonal $\operatorname{Diag}_{J}$ and let $N:=\{1, \ldots, n\}$. To simplify the notation, define $s(x)=n+1$ if $x \geq 0$ and $s(x)=n+2$ if $x<0$.

(1) $n+1, n+2 \in J$.

- For $\alpha \in\{n+1, n+2\}$ and $i \in J \cap N$, we substitute $\left(x_{\alpha}-x_{i}\right) \mapsto\left(x_{\alpha}-x_{i}\right)$.

- For $i \notin J \cap N$, we substitute $\left(x_{\alpha}-x_{i}\right) \mapsto\left(x_{\alpha}-x_{i}-b_{i}+t\right)$.

The initial term as a polynomial in $x_{i} \mathrm{~s}$ is then

$$
\begin{aligned}
& \prod_{i \in J \cap N}\left(x_{s\left(a_{i}\right)}-x_{i}\right)^{\left|a_{i}\right|} \prod_{i \in J^{c} \cap N}\left(t-b_{i}\right)^{\left|a_{i}\right|} \\
& \quad-\prod_{i \in J \cap N}\left(x_{s\left(-a_{i}\right)}-x_{i}\right)^{\left|a_{i}\right|} \prod_{i \in J^{c} \cap N}\left(t-b_{i}\right)^{\left|a_{i}\right|} .
\end{aligned}
$$

If $|J| \geq 3$ and $J \cap N$ is not contained in the set

$$
A_{0}:=\left\{i \mid a_{i}=0\right\},
$$

then the summed terms have distinct prime factors, so (21) is nonzero. Hence,

$$
k_{J}=\sum_{i \in J}\left|a_{i}\right|
$$

for $J$ containing some $i \in N$ with $a_{i} \neq 0$.

If $J \cap N \subset A_{0}$, then (21) is indeed zero, but the entire polynomial will be the same as that obtained via the requisite substitution for computation of the 
multiplicity of $F$ along $\operatorname{Diag}_{\{n+1, n+2\}}$. This particular substitution results in a coefficient of $x_{n+1}$ given by

$$
\begin{aligned}
\sum_{a_{i}>0}\left|a_{i}\right|\left(t-b_{i}\right)^{\left|a_{i}\right|-1} \prod_{j \neq i}\left(t-b_{j}\right)^{\left|a_{j}\right|} & \\
& -\sum_{a_{i}<0}\left|a_{i}\right|\left(t-b_{i}\right)^{\left|a_{i}\right|-1} \prod_{j \neq i}\left(t-b_{j}\right)^{\left|a_{j}\right|},
\end{aligned}
$$

which is nonzero since the summands have pairwise distinct prime factors. This shows that $k_{J}=1$ for $J \cap N \subset A_{0}$.

In particular, the multiplicity of our equation along $V\left(x_{n+1}-x_{n+2}\right)$ is 1 . This proves part of Claim 4.6:

$$
\max \left\{m \mid\left(x_{n+1}-x_{n+2}\right)^{m} \text { divides } F\right\}=1 .
$$

(2) $n+1, n+2 \notin J$.

- For $i \in J$, we substitute $\left(x_{\alpha}-x_{i}\right) \mapsto\left(x_{\alpha}-x_{i}-t+b_{\alpha}\right)$.

- For $i \in N \backslash J$, we substitute $\left(x_{\alpha}-x_{i}\right) \mapsto\left(x_{\alpha}-x_{i}-b_{i}+b_{\alpha}\right)$.

The constant term of the resulting polynomial in $x_{i}$ is

$$
\begin{aligned}
\prod_{i \in J}(t & \left.-b_{s\left(a_{i}\right)}\right)^{\left|a_{i}\right|} \prod_{i \notin J}\left(b_{i}-b_{s\left(a_{i}\right)}\right)^{\left|a_{i}\right|} \\
& -\prod_{i \in J}\left(t-b_{s\left(-a_{i}\right)}\right)^{\left|a_{i}\right|} \prod_{i \notin J}\left(b_{i}-b_{s\left(-a_{i}\right)}\right)^{\left|a_{i}\right|} .
\end{aligned}
$$

If there is an $i \in N \backslash J$ with $a_{i} \neq 0$, then we necessarily have a monomial $\left(b_{i}-b_{s}\left(a_{i}\right)\right)$ dividing one term but not the other, and the difference is nonzero. Hence, $N \backslash J \not \subset A_{0}$ implies that $k_{J}=0$. Now suppose that $a_{i}=0$ for all $i \in N \backslash J$. In this case, (22) is zero. Let $r=\sum_{a_{i}>0}\left|a_{i}\right|=\sum_{a_{i}<0}\left|a_{i}\right|$. The next lowest term as a polynomial in $x_{i}$ includes the summand

$$
\left[\left(t-b_{n+1}\right)^{r-1}\left(t-b_{n+2}\right)^{r}-\left(t-b_{n+1}\right)^{r}\left(t-b_{n+2}\right)^{r-1}\right]\left(\sum_{\substack{i \leq n \\ a_{i}>0}} x_{i}\right) .
$$

There are other degree 1 contributions, but these do not involve $x_{i}$ for $i \leq n$ and $a_{i}>0$, so to conclude that $k_{\{1, \ldots, n\}}=1$, it suffices to note that (23) is nonzero. This shows that

$$
\begin{array}{lll}
k_{J}=1 & \text { if } N \backslash J \subset\left\{i \mid a_{i}=0\right\} & \text { and } \\
k_{J}=0 & \text { otherwise. }
\end{array}
$$

In particular, if all $a_{i}$ are nonzero, then $k_{J} \neq 0$ if and only if $J=\{1, \ldots, n\}$, in which case $k_{J}=1$.

(3) $|\{n+1, n+2\} \cap J|=1$. Without loss of generality, assume that $n+1 \in J$ and $n+2 \notin J$; the argument is symmetric.

- For $i \in N \cap J$, we substitute $\left(x_{n+1}-x_{i}\right) \mapsto\left(x_{n+1}-x_{i}\right)$ and $\left(x_{n+2}-x_{i}\right) \mapsto$ $\left(x_{n+2}-x_{i}-t+b_{n+2}\right)$.

- For $i \in N \backslash J$, we substitute $\left(x_{n+1}-x_{i}\right) \mapsto\left(x_{n+1}-x_{i}-b_{i}+t\right)$ and $\left(x_{n+2}-\right.$ $\left.x_{i}\right) \mapsto\left(x_{n+2}-x_{i}-b_{i}+b_{n+2}\right)$. 
Define $h_{j, i}=\left(x_{n+j}-x_{i}\right)$ for $j \in\{1,2\}$ and $1 \leq i \leq n$. With this notation, substituting gives $F=$

$$
\begin{aligned}
\prod_{\substack{i \in J \\
a_{i}>0}}\left(h_{1, i}\right)^{\left|a_{i}\right|} \prod_{\substack{i \notin J \\
a_{i}>0}}\left(h_{1, i}-b_{i}+t\right)^{\left|a_{i}\right|} \prod_{\substack{i \in J \\
a_{i}<0}}\left(h_{2, i}-t+b_{n+2}\right)^{\left|a_{i}\right|} \\
\quad \times \prod_{\substack{i \notin J \\
a_{i}<0}}\left(h_{2, i}-b_{i}+b_{n+2}\right)^{\left|a_{i}\right|} \\
\quad-\prod_{\substack{i \in J \\
a_{i}<0}}\left(h_{1, i}\right)^{\left|a_{i}\right|} \prod_{\substack{i \notin J \\
a_{i}<0}}\left(h_{1, i}-b_{i}+t\right)^{\left|a_{i}\right|} \prod_{\substack{i \in J \\
a_{i}>0}}\left(h_{2, i}-t+b_{n+2}\right)^{\left|a_{i}\right|} \\
\quad \times \prod_{\substack{i \notin J \\
a_{i}>0}}\left(h_{2, i}-b_{i}+b_{n+2}\right)^{\left|a_{i}\right|} .
\end{aligned}
$$

The initial term of the expanded expression is

$$
\begin{aligned}
& \prod_{\substack{i \in J \\
a_{i}>0}}\left(x_{n+1}-x_{i}\right)^{\left|a_{i}\right|} \prod_{\substack{i \notin J \\
a_{i}>0}}\left(t-b_{i}\right)^{\left|a_{i}\right|} \prod_{\substack{i \in J \\
a_{i}<0}}\left(b_{n+2}-t\right)^{\left|a_{i}\right|} \prod_{\substack{i \notin J \\
a_{i}<0}}\left(b_{n+2}-b_{i}\right)^{\left|a_{i}\right|} \\
& \quad-\prod_{\substack{i \in J \\
a_{i}<0}}\left(x_{n+1}-x_{i}\right)^{\left|a_{i}\right|} \prod_{\substack{i \notin J \\
a_{i}<0}}\left(t-b_{i}\right)^{\left|a_{i}\right|} \prod_{\substack{i \in J \\
a_{i}>0}}\left(b_{n+2}-t\right)^{\left|a_{i}\right|} \\
& \quad \times \prod_{\substack{i \notin J \\
a_{i}>0}}\left(b_{n+2}-b_{i}\right)^{\left|a_{i}\right|} .
\end{aligned}
$$

The two terms comprising (25) necessarily have distinct factors regardless of the relationship between $A_{0}$ and $J$, so that the difference is nonzero. Hence,

$$
k_{J}=\min \left\{\sum_{\substack{a_{i} \geq 0 \\ i \in J}}\left|a_{i}\right|, \sum_{\substack{a_{i} \leq 0 \\ i \in J}}\left|a_{i}\right|\right\} .
$$

These formulae do not quite give the class of the divisor $\Lambda_{\mathbf{a}}$. Assuming Claim 4.6, the actual equation specifying $\Lambda_{\mathbf{a}}$ is $F /\left(x_{n+1}-x_{n+2}\right)$. Hence, the relevant multiplicities giving class coefficients are computed by subtracting the multiplicity of $\left(x_{n+1}-x_{n+2}\right)$ along $\operatorname{Diag}_{J}$ from each computed $k_{J}$. Our formulae will therefore be as follows:

(1) If $n+1, n+2 \in J$, substituting to compute the multiplicity along $\operatorname{Diag}_{J}$ gives $\left(x_{n+1}-x_{n+2}\right) \mapsto\left(x_{n+1}-x_{n+2}\right)$, so the multiplicity is 1 . Hence, defining $M=\{1, \ldots, n+2\}$,

$$
m_{M \backslash J}=k_{J}-1=\left(\sum_{i \in J}\left|a_{i}\right|\right)-1
$$

for $J \cap N \not \subset\left\{i \mid a_{i}=0\right\}$, and

$$
m_{M \backslash J}=0
$$

for $J \cap N \subset\left\{i \mid a_{i}=0\right\}$. 
(2) If $n+1, n+2 \notin J$, then we substitute $\left(x_{n+1}-x_{n+2}\right) \mapsto\left(x_{n+1}-x_{n+2}+\right.$ $\left.b_{n+1}-b_{n+2}\right)$, which shows that the multiplicity of $x_{n+1}-x_{n+2}$ is zero along $\operatorname{Diag}_{J}$. Hence,

$$
m_{M \backslash J}=0
$$

unless $M \backslash J \subset A_{0}$, in which case

$$
m_{M \backslash J}=k_{J}=1 .
$$

(3) $|\{n+1, n+2\} \cap J|=1$. Evidently, the multiplicity of $\left(x_{n+1}-x_{n+2}\right)$ here is also zero, and

$$
m_{N \backslash J}=k_{J}=\min \left\{\sum_{\substack{i \in J \\ a_{i} \geq 0}}\left|a_{i}\right|, \sum_{\substack{i \in J \\ a_{i} \leq 0}}\left|a_{i}\right|\right\} .
$$

Reformulating (1)-(3) gives the theorem.

It remains to complete the proof of Claim 4.6. We have already noted that $\left(x_{n+1}-x_{n+2}\right)^{k}$ divides $F$ if and only if $k=1$. To see that no other $\left(x_{i}-x_{j}\right)$ divides $F$ for $i \neq j$ and $i, j \leq n$, recall that $F$ has multiplicity zero along each partial diagonal $V\left(x_{i}-x_{j}\right)$ by (24). By inspection of (20) it is evident that neither $\left(x_{n+1}-x_{j}\right)$ nor $\left(x_{n+2}-x_{j}\right)$ can divide $F$ for $j \leq n$.

EXAmPLE 4.7. Let $D_{k}:=\Lambda_{(k, 1,-1,-1, \ldots,-1)} \subset \bar{M}_{0, k+5}$. Let $K=\{k+4, k+5\}$. We apply the formulas from Corollary 4.5 to compute the class of $D_{k}$ with respect to the Kapranov basis for $\bar{M}_{0, k+5}$ using index 1 . Note that, in our case,

$$
\sum_{\substack{1 \leq i \leq k+3 \\ i \neq 1}}\left|a_{i}\right|-1=\left(\sum_{2 \leq i \leq k+3}(1)\right)-1=k+1,
$$

and for $K \cap I=\emptyset$,

$$
\sum_{i \notin I \cup\{1\}}\left|a_{i}\right|-1=|\{2, \ldots, k+3\}-I|-1=k+2-|I|-1=k+1-|I| .
$$

For $|K \cap I|=1$, the coefficient is

$$
\min \left\{\sum_{\substack{0 \leq a_{i} \\ i \notin I \cup\{1\}}}\left|a_{i}\right|, \sum_{\substack{0 \geq a_{i} \\ i \notin I \cup\{1\}}}\left|a_{i}\right|\right\}
$$

If $2 \in I$, then the minimum is zero; if not, the minimum is always one since cardinality considerations show that

$$
\left\{i \mid a_{i} \leq 0\right\} \not \subset I .
$$

Hence, we have that

$$
D_{k} \sim(k+1) H-\sum_{i=1}^{k}\left(\sum_{\substack{K \cap I=\emptyset \\|I|=i}}(k+1-i) E_{I}\right)-\sum_{\substack{|K \cap I|=1 \\ 2 \notin I}} E_{I}
$$


ExAmPle 4.8. Consider the $(k+1)$-tuple $(k,-1,-1,-1, \ldots,-1)$, which gives a divisor on $\bar{M}_{0, k+3}$. Note that, by Theorem 4.10 , this divisor can be obtained by intersecting $D_{k}$ from Example 4.7 with the boundary where the first two markings "collide". Using Corollary 4.5, we compute the class of $L_{k}:=\Lambda_{(k,-1, \ldots,-1)}$ with respect to the index 1 Kapranov basis:

$$
L_{k} \sim(k-1) H-\sum_{i=1}^{k-1} \sum_{\substack{|I|=i \\ I \subseteq\{2, \ldots, k+1\}}}(k-1-i) E_{I} .
$$

Note that all $E_{I}$ with $k+2 \in I$ or $k+3 \in I$ do not contribute to the class of $L_{k}$.

We return to general results on Chen-Coskun divisors. The next theorem relates certain Chen-Coskun divisors to spherical hypertree divisors (defined in Section 3).

THEOREM 4.9. If $\mathbf{a}=(1,1, \ldots,-1,-1, \ldots)$ is a $2 k$-tuple with $\sum a_{i}=0$, then $\Lambda_{\mathbf{a}}=D_{\Gamma}$ where $\Gamma$ is the spherical hypertree divisor associated to a bipyramidal bicolored spherical triangulation with $2 k$ triangles.

Proof. Since $D_{\Gamma}$ and $\Lambda_{\mathbf{a}}$ are irreducible, it suffices to show that $D_{\Gamma} \cap M_{0, n} \subset \Lambda_{\mathbf{a}}$. For this, we appeal to a characterization of spherical bipyramid hypertree divisors given in [CT, 9.5].

Let $\Gamma$ be the spherical bipyramid divisor on $n=2 k+2$ vertices. Then there is a partition of $1, \ldots, n$ into subsets $X, Y, Z$ with $|X|=|Y|=k$ and $|Z|=2$, where the indices in $Z$ correspond to "poles" of the bipyramid, and those in $X$ and $Y$ are alternating points on the "equator" (see Figure 4$)$.

Assume that $X=\{1, \ldots, k\}, Y=\{k+1, \ldots, 2 k\}, Z=\{2 k+1,2 k+2\}$. Consider the embedding $\eta$ of $\left[\mathbb{P}^{1} ; p_{1}, \ldots, p_{n}\right]$ into $\mathbb{P}^{k}$ as a rational normal curve degree $k$; let $q_{i}=\eta\left(p_{i}\right)$, and

$$
\begin{aligned}
L & =\left\langle q_{i}\right\rangle_{i \in Z}, \\
\tilde{X} & =\left\langle q_{i}\right\rangle_{i \in X}, \\
\tilde{Y} & =\left\langle q_{i}\right\rangle_{i \in Y} .
\end{aligned}
$$

Castravet and Tevelev show that $D_{\Gamma}$ consists of $\left[\mathbb{P}^{1} ; p_{1}, \ldots, p_{n}\right]$ such that

$$
L \cap \tilde{X} \cap \tilde{Y} \neq \varnothing \text {. }
$$

Fix a representative of marked points $p_{i}$ for an arbitrary element in $D_{\Gamma} \cap M_{0, n}$. The function with zeros of order one at $p_{i}$ for $i \in X$ and poles of order one at $p_{i}$ for $i \in Y$ is given by $h=\eta^{*}\left(h_{1} / h_{2}\right)$, where $h_{1}$ is a linear equation of $\tilde{X}$, and $h_{2}$ one of $\tilde{Y}$. Let $q_{n+i}=\left[a_{1 i}: \cdots: a_{k i}\right]$ for $i=1,2$. Then (27) implies that, for some elements $s$ and $t$ in the base field,

$$
h_{j}\left(\left[s a_{11}+t a_{12}: \cdots: s a_{k 1}+t a_{k 2}\right]\right)=0
$$

for $j=1$ or $j=2$. This, together with linearity, implies that

$$
\frac{h_{1}}{h_{2}}\left(q_{n+1}\right)=\frac{h_{1}}{h_{2}}\left(q_{n+2}\right),
$$




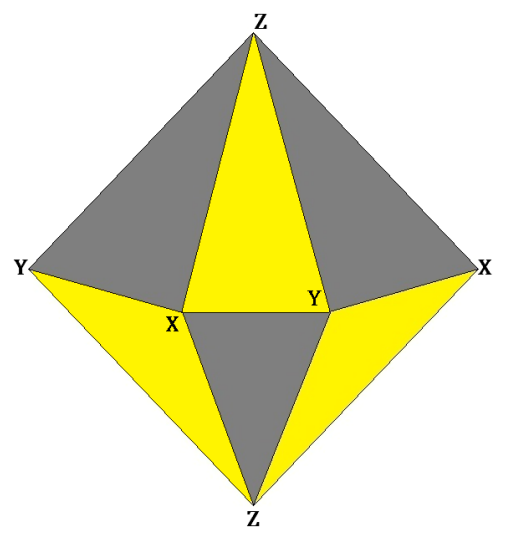

Figure 4 Spherical pyramidal triangulation with subsets $X, Y$, and $Z$ indicated

so that

$$
\eta^{*} \frac{h_{1}}{h_{2}}\left(x_{n+1}\right)=\eta^{*} \frac{h_{1}}{h_{2}}\left(q_{n+2}\right) .
$$

Hence, $\operatorname{div}(h)=p_{1}+\cdots+p_{k}-p_{k+1}-\cdots-p_{2 k}$ and $h\left(p_{2 k+1}\right)=h\left(p_{2 k+2}\right)$, so $h$ witnesses that $\left[\mathbb{P} ; p_{1}, \ldots, p_{n}\right] \in \Lambda_{(1, \ldots, 1,-1, \ldots,-1)}$.

The next theorem describes how any Chen-Coskun divisor arises from intersections of a "universal divisor" of the form $\Lambda_{(1,1, \ldots,-1,-1, \ldots)}$ with boundary divisors. Together with the previous result, this gives a relationship between Chen-Coskun divisors and hypertree divisors: all Chen-Coskun divisors are obtained by a sequence of restrictions of a bipyramidal spherical hypertree divisor.

Note that, in an attempt to clarify the proof of the theorem, we use labels $0, \ldots, n+2$ for markings on $\bar{M}_{0, n+3}$ and markings $1, \ldots, n+2$ on $\bar{M}_{0, n+2}$.

THEOREM 4.10. Let $\mathbf{a}=\left(a_{0}, \ldots, a_{n}\right) \in \mathbb{Z}^{n+1}$ be such that $\sum_{i} a_{i}=0$ and $\operatorname{gcd}\left(a_{0}, \ldots, a_{n}\right)=\operatorname{gcd}\left(a_{0}+a_{1}, a_{2}, \ldots, a_{n-1}, a_{n}\right)=1$. Define $\mathbf{b}=\left(a_{0}+a_{1}, \ldots\right.$, $\left.a_{n-1}, a_{n}\right) \in \mathbb{Z}^{n}$. Then $\Lambda_{\mathbf{a}} \cap \delta_{\{0,1\}}=\Lambda_{\mathbf{b}}$ as a divisor on $\delta_{\{0,1\}} \simeq \bar{M}_{0, n+2}$.

Proof. Consider the following diagram:

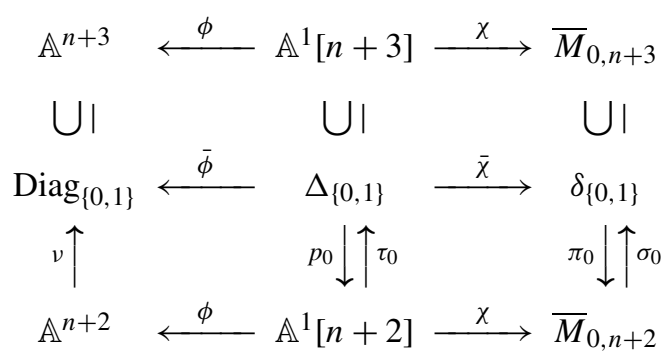

The maps $\phi$ and $\chi$ are as in (1). The maps $\bar{\phi}$ and $\bar{\chi}$ are restrictions of these to the indicated subsets. The isomorphisms of $\delta_{\{0,1\}}$ with $\bar{M}_{0, n+2}$ and $\Delta_{\{0,1\}}$ with 
$\mathbb{A}^{1}[n+2]$ are restrictions of the index 0 forgetful morphisms; the inverse maps are given by index 0 sections, $\sigma_{0}$ and $\tau_{0}$. The isomorphism $v$ is defined by

$$
\left(x_{1}, \ldots, x_{n}, x_{n+1}, x_{n+2}\right) \mapsto\left(x_{1}, x_{1}, \ldots, x_{n}, x_{n+1}, x_{n+2}\right) .
$$

(That is, the map "repeats the first index".)

The commutativity of the lower left rectangle follows from the fact that the iterated blow-up defining $\mathbb{A}^{1}[n+3]$ restricts to an iterated blow-up of the subspace $\operatorname{Diag}_{\{0,1\}}$; this coincides with the Fulton-MacPherson construction when $\operatorname{Diag}_{\{0,1\}}$ is naturally identified with $\mathbb{A}^{n+2}$. The commutativity of the lower right rectangle is immediate from that of (1).

Define

$$
\begin{aligned}
\partial & =\bigcup_{\substack{|I| \geq 2 \\
I \neq\{0,1\}}} \delta_{I}, \\
V & =\bigcup_{\{i, j\} \neq\{0,1\}} \operatorname{Diag}_{\{i, j\}}, \\
\delta_{\{0,1\}}^{0} & =\delta_{\{0,1\} \backslash \partial,} \\
A & =\mathbb{A}^{n+3} \backslash V, \\
M & =M_{0, n+3} \cup \delta_{\{0,1\}}^{0}, \\
B & =\mathbb{A}^{n+2} \backslash\{\text { diagonals }\} .
\end{aligned}
$$

Let $\Lambda_{\mathbf{a}}^{0}=\Lambda_{\mathbf{a}} \cap M$ and $\Lambda_{\mathbf{b}}^{0}=\Lambda_{\mathbf{b}} \cap M_{0, n+2}$. We will show that $\Lambda_{\mathbf{a}}^{0} \cap \delta_{\{0,1\}}=\Lambda_{\mathbf{b}}^{0}$, so $\Lambda_{\mathbf{a}} \cap \delta_{\{0,1\}}$ and $\Lambda_{\mathbf{b}}$ can differ only by boundary divisors of $\delta_{\{0,1\}} \simeq \bar{M}_{0, n+2}$. Given this, for the equality of the divisors, it will suffice to show that they have the same classes.

Let $F$ be the polynomial in $k\left[x_{0}, \ldots, x_{n+2}\right]$ specifying $\Lambda_{\mathbf{a}}$; the form of $F$ is given in (16). Since $\Lambda_{\mathbf{a}}^{0} \cap \delta_{\{0,1\}}=\sigma_{0}^{-1}\left(\Lambda_{\mathbf{a}} \cap M\right)$ and the diagram commutes, we have that

$$
\begin{aligned}
\chi^{-1}\left(\Lambda_{\mathbf{a}}^{0} \cap \delta_{\{0,1\}}\right) & =\chi^{-1}\left(\sigma_{0}^{-1}\left(\Lambda_{\mathbf{a}} \cap M\right)\right) \\
& =\tau_{0}^{-1}\left(\chi^{-1}\left(\Lambda_{\mathbf{a}} \cap M\right)\right) \\
& =\tau_{0}^{-1}\left(\phi_{*}^{-1}(V(F) \cap A)\right) \\
& =\phi_{*}^{-1}\left(v^{-1}(V(F)) \cap B\right)=\phi^{-1}\left(V\left(v^{*} F\right) \cap B\right) .
\end{aligned}
$$

But $h^{*} F$ is simply $F\left(x_{0}, \ldots, x_{n}, x_{n+1}, x_{n+2}\right)$ with $x_{1}$ substituted for $x_{0}$. If both $a_{1}$ and $a_{0}$ are nonnegative, we quite literally add exponents and obtain the equation $G\left(x_{1}, \ldots, x_{n+2}\right)$ that specifies $\Lambda_{\mathbf{b}}$ on $\bar{M}_{0, n+2}$. If $a_{0} \geq 0$ and $a_{1}<0$, then the substitution yields

$$
h^{*} F=\left(\left(x_{n+1}-x_{1}\right)\left(x_{n+2}-x_{1}\right)\right)^{\min \left\{\left|a_{0}\right|,\left|a_{1}\right|\right\}} G .
$$

Since the factors $\left(x_{n+1}-x_{1}\right)$ and $\left(x_{n+2}-x_{1}\right)$ contribute boundaries, the divisor specified by $h^{*} F$ coincides with that specified by $G$ on $M_{0, n+2}$. Hence, $\Lambda_{\mathbf{a}} \cap$ $\delta_{\{0,1\}}$ and $\Lambda_{\mathbf{b}}$ are specified by the same polynomial on the interior of $\bar{M}_{0, n+2}$, as claimed. 
We now show that the classes of $\Lambda_{\mathbf{a}} \cap \delta_{\{0,1\}}$ and $\Lambda_{\mathbf{b}}$ are the same. Note that $H$ and $\delta_{I}$ for $|I \cap\{0,1\}|=1$ restrict to the zero class on $\delta_{\{0,1\}}$. For $\{0,1\} \subsetneq I$, we have that $\delta_{I} \cap \Lambda_{\mathbf{a}}=\delta_{I \backslash\{0\}}$ on $\delta_{\{0,1\}}$ naturally identified with $\bar{M}_{0, n+2}$ with markings $1, \ldots, n+2$. The divisor $\delta_{\{0,1\}}$ restricts to $-H$ on $\delta_{\{0,1\}}$, the negative of a Kapranov hyperplane class with respect to the index 1 Kapranov basis. Applying this to the class of $\Lambda_{\mathbf{a}}$, we see that if

$$
\Lambda_{\mathbf{a}} \sim d H-\sum m_{I} E_{I}
$$

then

$$
\Lambda_{\mathbf{a}} \cap \delta_{\{1\}} \sim m_{\{1\}} H-\sum_{\{1\} \subsetneq I} m_{I} \delta_{I} .
$$

Note that $1 \leq|I| \leq n-1$, so that $1 \leq|I \backslash\{1\}| \leq n-2$ for $\{1\} \subsetneq I$ and $\delta_{I}=$ $E_{I \backslash\{1\}}$ on $\bar{M}_{0, n+2}$ using the Kapranov basis in index 1 . Hence, to show that $\Lambda_{\mathbf{a}} \cap$ $\delta_{\{0,1\}}$ has the same class as $\Lambda_{\mathbf{b}}$, we must verify that if $\Lambda_{\mathbf{b}}=g H-\sum n_{I} E_{I}$, then $m_{\{1\}}=g$ and $n_{I \backslash\{1\}}=m_{I}$.

By Corollary 4.4 we see that $g=\sum_{i \neq 1}\left|b_{i}\right|-1=\sum_{i \neq 0,1}\left|a_{i}\right|-1=m_{\{0,1\}}$, as desired. Moreover,

$$
\begin{aligned}
& |\{n+1, n+2\} \cap I|=|\{n+1, n+2\} \cap I \backslash\{1\}|, \\
& \left\{i \mid a_{i} \neq 0\right\} \subset I \quad \Longleftrightarrow \quad\left\{i \mid b_{i} \neq 0\right\} \subset I \backslash\{1\},
\end{aligned}
$$

and

$$
\left\{i \mid b_{i} \neq 0\right\} \subset\{2, \ldots, n\} \backslash I \quad\left\{\quad \Longleftrightarrow \quad\left\{i \mid a_{i} \neq 0\right\} \subset\{1, \ldots, n\} \backslash I .\right.
$$

This implies that the coefficient of $E_{I}$ in the class of $\Lambda_{\mathbf{a}}$ and of $E_{I \backslash\{1\}}$ in the class of $\Lambda_{\mathbf{b}}$ are computed using the same formula from Corollary 4.4. Noting that the formulas depend only on sums over the complements of $I \cup\{0\}$ and $I$, respectively, so that $m_{I}=n_{I \backslash\{1\}}$, as desired.

\section{Counterexample to the Castravet-Tevelev Conjecture}

In Example 4.7, we computed the class of $D_{k}=\Lambda_{(k, 1,-1, \ldots)}$ on $\bar{M}_{0, k+5}$ with respect to the index 1 Kapranov basis:

$$
\begin{gathered}
D_{k} \sim(k+1) H-\sum_{\substack{K \cap I=\emptyset \\
|I|=1}} k E_{I}-\sum_{\substack{K \cap I=\emptyset \\
|I|=2}}(k-1) E_{I}-\cdots \\
-\sum_{\substack{K \cap I=\emptyset \\
|I|=k}} E_{I}-\sum_{\substack{|K \cap I|=1 \\
2 \notin I}} E_{I},
\end{gathered}
$$

where we define $K=\{k+4, k+5\}$.

The divisor $D_{k}$ is evidently effective. For extremality, we appeal to the criterion given by Corollary 6.4: we construct an irreducible covering family of curves with $C \cdot D_{k}<0$. Define a Kapranov map $\psi_{1}$ from $\bar{M}_{0, k+5}$ to $\mathbb{P}^{k+2}$. Let $p_{2}, \ldots, p_{k+5}$ be the points in $\mathbb{P}^{k+2}$ such that $E_{I} \mapsto\left\langle p_{i}\right\rangle_{i \in I}$. Inspection of (29) shows that the image $S=\psi_{1}\left(D_{k}\right)$ is a hypersurface of degree $k+1$ with a point of multiplicity $k$ at each $p_{i}$ for $2 \leq i \leq k+3$. Moreover, we have $2 k+2$ codimension 2 subspaces 


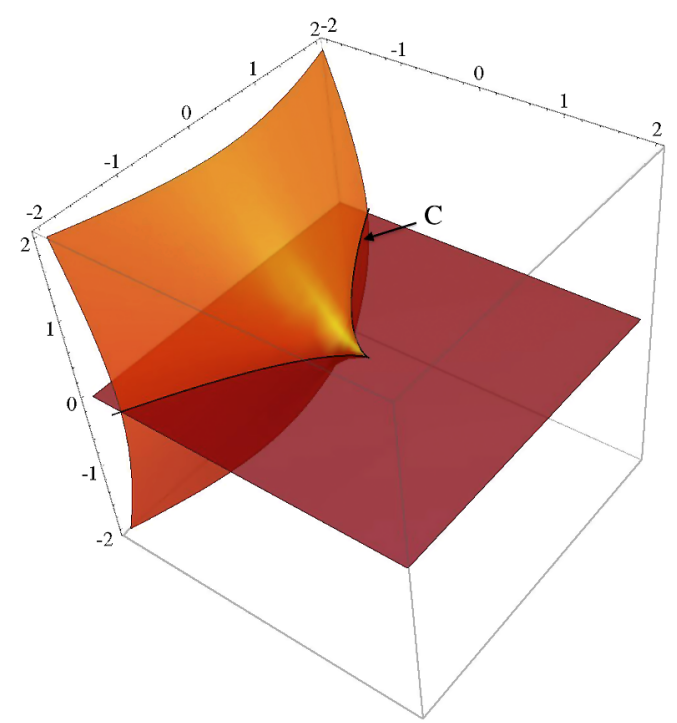

Figure 5 Schematic of the construction of an element $C$ in the covering family of curves $\mathcal{G}$ for $S \subset \mathbb{P}^{k+2}$

$\left\langle p_{i}\right\rangle_{i \in J}$ for $|J|=k+1,|K \cap J|=1$, and $2 \notin J$ which are contained with multiplicity 1 . To see this last fact, note that there are $k+1$ subsets of $\{3, \ldots, k+3\}$ of size $k$, obtained by omitting a single index. Augmenting these subsets with either index $k+4$ or index $k+5$ gives $2 k+2$ codimension 2 spans as claimed.

In $\mathbb{P}^{k+2}$, consider the family of curves $\mathcal{G}$ obtained by intersecting a 2-plane through $p_{2}$ with $S$ (see Figure 5). Let $\mathcal{F}$ denote the covering family of $D_{k}$ obtained by taking proper transforms of curves in $\mathcal{G}$ with respect to $\psi_{1}$.

Lemma 5.1. A general curve $C$ in the covering family $\mathcal{F}$ of $D_{k}$ has intersection pairing -1 with $D_{k}$.

Proof. By construction, the image $\psi_{1}(C)$ in $\mathbb{P}^{k+2}$ intersects a hyperplane in $k+1$ points, passes through $p_{2}$ with multiplicity $k$, and transversally intersects the $2 k+2$ codimension 2 linear spans $\left\langle p_{i}\right\rangle_{i \in I}$ for $|I|=n+1,2 \notin I$, and $|I \cap\{k+4, k+5\}|=1$ that contribute to the class of $D_{k}$. Hence, $C \cdot D_{k}=$ $(k+1)(k+1)-(k)(k)-(1)(2 k+2)=k^{2}+2 k+1-k^{2}-2 k-2=-1$.

Lemma 5.2. A general curve $C$ in the covering family $\mathcal{F}$ of $D_{k}$ is irreducible.

Proof. Note that it suffices to prove that $\psi_{1}(C)$ is irreducible, that is, that a general curve in $\mathcal{G}$ is irreducible. Let $T$ be the union of all lines through $p_{2}$ that are contained in $S$. Note that $T$ must have codimension at least 2 since otherwise $S$ contains a codimension 1 cone over $p_{2}$ and by irreducibility $S$ itself is a cone over $p_{2}$. However, $p_{2}$ is a point of multiplicity one less than the degree of $S$, so this is a contradiction. 
If we consider the map $\pi: \mathbb{P}^{n+2} \backslash\left\{p_{2}\right\} \rightarrow \mathbb{P}^{n+1}$ that projects from the point $p_{2}$, then the image of $T$ is a subvariety of codimension at least 2 , and the image of a 2-plane through $p_{2}$ is a line. Hence, for a general 2-plane $h$ containing $p_{2}$, $\pi(h) \cap \pi(T)=\emptyset$. We can reformulate this statement as follows: for a general 2-plane $h \subset \mathbb{P}^{k+2}$ containing $p_{2}$, the curve $S \cap h$ contains no line through $p_{2}$.

Now, for a contradiction, suppose that the intersection of a general 2-plane with $S$ is reducible. Then the plane curve obtained via intersection is the union of a curve $g_{1}$ of degree $m_{1}$ and a curve $g_{2}$ of degree $m_{2}$ for $m_{1}, m_{2} \geq 1$. Without loss of generality, $p_{2}$ is a point of multiplicity $m_{1}$ on $g_{1}$. But then $g_{1}$ is a union of lines through $p_{2}$.

It is shown in the next section that the preceding two lemmas imply the following:

Corollary 5.3. For each $k, D_{k}$ generates an extremal ray of the effective cone of $\bar{M}_{0, k+5}$.

We now verify that $D_{k}$ is not linearly equivalent to a hypertree divisor or hypertree divisor pull-back for $k \geq 2$. To this end, consider the class of $\pi_{k+6}^{-1}\left(D_{k}\right) \subset \bar{M}_{0, k+6}$ with respect to the index $k+6$ Kapranov basis. By Theorem 4.2,

$$
\pi_{k+6}^{-1}\left(D_{k}\right) \sim(2 k+1) H-\cdots .
$$

From the proof of Theorem 3.1, a hypertree divisor on less than or equal to $t$ vertices is specified by a polynomial of degree at most $t-3$. Hence, given a hypertree divisor or hypertree divisor pull-back $D_{\Gamma} \subset \bar{M}_{0, k+5}$, we have that

$$
\pi_{k+6}^{-1}\left(D_{\Gamma}\right) \sim s H-\cdots
$$

where $s \leq k+2$. Evidently, $s=2 k+1$ is impossible unless $k=1$.

\section{Covering Families of Curves and Conditions for Extremality}

Results of this section will imply Corollary 5.3. In fact, a sufficient result for Corollary 5.3 is proved in [CC, 4.1]: Chen and Coskun show that if $D$ is an irreducible divisor and there exists an irreducible curve $C$ so that $C \cdot D<0$ and $D$ is covered by irreducible curves numerically equivalent to $C$, then $D$ generates an extremal ray of the pseudoeffective cone.

We prove a slightly stronger result: under the same hypotheses, $D$ generates an "edge" of the pseudoeffective cone. Roughly, this means that $D$ is extremal and additionally the boundary of the pseudoeffective cone is not rounded near the ray generated by $D$. The proof, completed in Corollary 6.4 , follows from two lemmas of convex geometry. We first set up some notation; throughout we use standard Euclidean notions of distance, boundedness, et cetera on $\mathbb{R}^{N}$ with usual coordinates. 
Given a convex cone $X \subset \mathbb{R}^{N}$, we say that $v \in X$ is an edge for $X$ if there exist linear functions $h_{1}, \ldots, h_{N-1}$ such that

$$
\bigcap_{i=1}^{N-1}\left\{h_{i}=0\right\}=\langle v\rangle
$$

and

$$
X \subset \bigcap_{i=1}^{N-1}\left\{h_{i} \geq 0\right\} .
$$

We say that $v \in X$ is extremal if $v=a_{1} w_{1}+a_{2} w_{2}$ for $a_{1}, a_{2} \geq 0$ and $w_{1}, w_{2} \in X$ implies that $w_{1}$ and $w_{2}$ are proportional to $v$.

Lemma 6.1. If $X$ is a convex cone in $\mathbb{R}^{N}$ and $v \in X$ is an edge, then $v$ is extremal.

Proof. If $v=a_{1} w_{1}+a_{2} w_{2}$, then $0=h_{i}(x)=a_{1} h_{i}\left(w_{1}\right)+a_{2} h_{i}\left(w_{2}\right)$ for each $1 \leq$ $i \leq N-1$. Since $w_{i} \in X, h_{i}\left(w_{i}\right) \geq 0$ for each $i$ by (31) and since $a_{j} \geq 0$, we must have $h_{i}\left(w_{1}\right)=h_{i}\left(w_{2}\right)=0$ for all $i$. Hence, by (30), $w_{j} \in\langle v\rangle$, as desired.

Given a collection of points $V \subset \mathbb{R}^{N}$, let $\mathrm{C}(V)$ denote the closure of the convex hull of all nonnegative multiples of elements in $V$. This is, in particular, a closed convex cone in $\mathbb{R}^{N}$.

Lemma 6.2. Given $V \subset \mathbb{R}^{N}$ and $v \in V$, suppose that

(a) There exists a linear function $\sigma$ such that $\sigma(x)<0$ for $x \in V$ if and only if $x=v$;

(b) There exists an affine hyperplane $0 \notin H \subset \mathbb{R}^{N}$ with $H \cap \mathrm{C}(V)$ nonempty and bounded.

Then $v$ is an edge of $\mathrm{C}(V)$. Moreover, $v$ is extremal.

Proof. We assume throughout that generating set $V$ contains two points that are not multiples of each other, since the lemma is immediate when $V=\{v\}$.

For any $x \in V$, some positive multiple of $x$ lies in $H$. To see this, note that without loss of generality $H=\{z \mid g(z)=1\}$ for some linear function $g$. If $\lambda x \notin$ $H$ for all $\lambda \geq 0$, we must have $g(x)=b \leq 0$. Choose any $y \in H \cap C(V)$ not a scalar multiple of $x$. Such a $y$ exists by the assumption that $V$ contains a pair of linearly independent points. If $b=0$, then $y+\lambda x \in \mathrm{C}(V) \cap H$ for all $\lambda>0$, which contradicts the boundedness of $H \cap \mathrm{C}(V)$.

If $b<0$, let $\lambda_{1}>1$ and $\lambda_{2}=\frac{\lambda_{1}-1}{|b|}>0$. Then $\lambda_{1} y+\lambda_{2} x \in H \cap \mathrm{C}(V)$ since $\lambda_{1}, \lambda_{2}>0$ and $g\left(\lambda_{1} y+\lambda_{2} x\right)=1$. Since $x$ and $y$ are linearly independent and $\lambda_{2} \rightarrow \infty$ as $\lambda_{1} \rightarrow \infty$, this gives an unbounded sequence in $H \cap \mathrm{C}(V)$, again contradicting (b). Let $K:=\mathrm{C}(V) \cap H$. We have shown that $K$ is closed, bounded, and convex and that

$$
\mathrm{C}(V)=\{\lambda x \mid x \in K, \lambda \geq 0\} .
$$

These observations will be used later.

Now let $T$ denote the subspace $\{y \mid \sigma(y)=0\} \subset \mathbb{R}^{N}$, where $\sigma$ is supplied by (a). 
Claim 6.3. There exists a basis $\left\{v, x_{1}, \ldots, x_{N-1}\right\}$ for $\mathbb{R}^{N}$ such that if we denote by $h_{i}$ the coordinate function naturally associated to the element $x_{i}$ of the basis $\left\{v, x_{i}\right\}$, then:

- $x_{i} \in T$ for $1 \leq i \leq N-1$.

- $K \cap T \subset \bigcap_{i=1}^{N-1}\left\{h_{i} \geq 0\right\}$.

Given the claim, since $\mathrm{C}(V) \cap T$ consists of nonnegative multiples of elements of $K \cap T$, it follows that $\mathrm{C}(V) \cap T$ also lies in this intersection of half-spaces. With notation as in the claim, we have that

$$
V \subset \bigcap_{i=1}^{N-1}\left\{h_{i} \geq 0\right\} .
$$

Indeed, let $y \in V \backslash\{v\}$. Then

$$
y=-\alpha v+\sum_{i} a_{i} x_{i}
$$

for some uniquely determined coefficients $\alpha, a_{i}$. Moreover,

$$
\sigma(y)=-\alpha \sigma(v) \geq 0
$$

by assumption (a). Since $\sigma(v)<0$, we must have $\alpha \geq 0$. Then $\alpha v+y=\sum_{i} a_{i} x_{i} \in$ $\mathrm{C}(V) \cap T$, so that $a_{i} \geq 0$ by choice of the basis $x_{i}$.

This shows that

$$
\mathrm{C}(V) \subset \bigcap_{i=1}^{N-1}\left\{h_{i} \geq 0\right\}
$$

and

$$
\bigcap_{i=1}^{N-1}\left\{h_{i}=0\right\}=\langle v\rangle,
$$

so that $v$ is an edge for $\mathrm{C}(V)$, as desired.

To prove Claim 6.3, let $K_{0}:=K \cap T$. Since $K=\mathrm{C}(V) \cap H$, we have that

$$
K_{0} \subset H \cap T=\{x \in T \mid g(x)=1\},
$$

where, as before, $g$ is a linear function on $\mathbb{R}^{N}$, so that $H=\left\{x \in \mathbb{R}^{N} \mid g(x)=1\right\}$. This subset is nonempty since $\sigma(v)<0$ but $\sigma(x) \geq 0$ for some $x \in V$, so that we can find $x^{\prime} \in \mathrm{C}(V)$ with $\sigma\left(x^{\prime}\right)=0$. As argued previously, some positive multiple of $x^{\prime}$ lies in $H$ and therefore in $K_{0}$.

If we take $T_{0}$ to be the $(N-2)$-dimensional subspace of $T$ where $g$ vanishes, we have $T_{0} \cap K_{0}=\emptyset$. Let $x_{1}^{\prime}$ be a normal vector to $T_{0}$ with $g(x)>0$. Let $x_{2}^{\prime}, \ldots, x_{N-1}^{\prime}$ be a basis for $T_{0}$. Then let $h_{i}^{\prime}$ denote the coordinate functions associated to this basis, and by the boundedness of $K_{0}$ we have that for each $i$, $h_{i}^{\prime}\left(K_{0}\right) \subset\left[a_{i}, b_{i}\right]$ for some finite $a_{i}, b_{i}$. Note that by assumption $a_{1}>0$. Now 
define new coordinates by $x_{i}=x_{i}^{\prime}$ for $i \geq 2$ and

$$
x_{1}=x_{1}^{\prime}+\sum_{\substack{i=2 \\ a_{i}<0}}^{N-1} \frac{a_{i}}{a_{1}} x_{i}^{\prime} .
$$

If $y \in K_{0}$, then $y=\lambda_{1} x_{1}^{\prime}+\sum_{i=2}^{N-1} \lambda_{i} x_{i}^{\prime}$ for $\lambda_{i} \geq a_{i}$. Substituting to express $y$ with respect to the basis $\left\{x_{i}\right\}$, we obtain

$$
y=\lambda_{1} x_{1}+\sum_{\substack{i=2 \\ a_{i}<0}}^{N-1}\left(-\frac{\lambda_{1}}{a_{1}} a_{i}+\lambda_{i}\right) x_{i}+\sum_{\substack{i=2 \\ a_{i} \geq 0}}^{N-1} \lambda_{i} x_{i} .
$$

All coordinates of vectors in $K_{0}$ are positive with respect to the new basis since $-\frac{\lambda_{1}}{a_{1}} a_{i}+\lambda_{i}>-\frac{\lambda_{1}}{a_{1}} a_{i}+a_{i}>0$ for $a_{i}<0$.

The second assertion of the lemma is immediate from Lemma 6.1.

Corollary 6.4. Given an irreducible effective divisor $D$ on a smooth projective variety $X$ and an irreducible covering family of curves $C$ such that $C \cdot D<0$, the divisor $D$ generates an edge of the effective cone and is therefore extremal.

Proof. If we let $H$ denote the class group of $X$ modulo numerical equivalence and identify $\mathrm{NS}(X)=H \otimes \mathbb{R}$ with $\mathbb{R}^{N}$ for suitable $N$, then $\overline{\operatorname{Eff}}(X)=\mathrm{C}(S)$ where $S \subset \mathrm{NS}(X)$ is the set of irreducible effective divisor classes.

For any irreducible divisor $D^{\prime}$, we may choose an irreducible curve $C^{\prime}$ in the family that does not lie in the intersection of the $D^{\prime}$ and $D$. Then $C^{\prime} \cdot D^{\prime} \geq 0$. This shows that (a) from Lemma 6.2 is satisfied. It is a well-known fact that the pseudoeffective cone has nonempty, bounded intersection with an appropriately chosen affine hyperplane, so (b) is also satisfied.

\section{Rigid Examples and Nonextremal Examples}

We say that a divisor $D$ is rigid if $h^{0}(k D)=\operatorname{dim} H^{0}\left(\mathcal{O}_{X}(k D)\right)=1$ for all $k \geq 1$. Before commencing with examples, we record the following:

LemMA 7.1. Suppose that $X$ is a smooth projective variety and $D \subset X$ is an irreducible effective divisor with a covering family $\mathcal{F}$ of irreducible curves such that $C \cdot D<0$ for $C \in \mathcal{F}$. Then $D$ is rigid.

Proof. See [CC, 4.1].

TheOREM 7.2. Given $\mathbf{a}=\left(a_{1}, 1, \ldots, 1,-1, \ldots,-1\right)$ with $a_{1}>1$, the divisor $\Lambda_{\mathbf{a}}$ on $\bar{M}_{0, N+2}$ is rigid. Here, $N=a_{1}+2 m+1$, and $m$ equals the number of positive 1 s appearing in the entries of $\mathbf{a}$.

Proof. We proceed by induction on $m$. If $m=1$, then $\Lambda_{\mathbf{a}}$ is one of the counterexamples provided in Section 5, and hence there exists an irreducible covering 
family of curves for the divisor with negative intersection pairing; Lemma 7.1 implies that $\Lambda_{\mathbf{a}}$ is rigid.

Now suppose that $m>1$. If $\mathbf{b}:=\left(\mathbf{a}_{\mathbf{1}}+\mathbf{1}, \mathbf{1}, \ldots, \mathbf{1}, \mathbf{1},-\mathbf{1},-\mathbf{1}, \ldots,-\mathbf{1}\right)$, then by Theorem 4.10 we have that $\Lambda_{\mathbf{a}} \cap \delta_{\{1,2\}} \simeq \Lambda_{\mathbf{b}}$ when $\delta_{\{1,2\}}$ is naturally identified with $\bar{M}_{0, N+1}$. By induction $\Lambda_{\mathbf{b}}$ is rigid. We have an exact sequence

$$
0 \longrightarrow \mathcal{O}_{\bar{M}_{0, N+2}}\left(k \Lambda_{\mathbf{a}}-\delta_{\{1,2\}}\right) \longrightarrow \mathcal{O}_{\bar{M}_{0, N+2}}\left(k \Lambda_{\mathbf{a}}\right) \longrightarrow \mathcal{O}_{\delta_{\{1,2\}}}\left(k \Lambda_{\mathbf{b}}\right) \longrightarrow 0,
$$

which gives a long exact sequence in cohomology

$$
\begin{aligned}
0 & \longrightarrow H^{0}\left(\mathcal{O}_{\bar{M}_{0, N+2}}\left(k \Lambda_{\mathbf{a}}-\delta_{\{1,2\}}\right)\right) \longrightarrow H^{0}\left(\mathcal{O}_{\bar{M}_{0, N+2}}\left(k \Lambda_{\mathbf{a}}\right)\right) \\
& \longrightarrow H^{0}\left(\mathcal{O}_{\delta_{\{1,2\}}}\left(k \Lambda_{\mathbf{b}}\right)\right) \longrightarrow \cdots .
\end{aligned}
$$

Since $\operatorname{dim} H^{0}\left(\mathcal{O}_{\delta_{\{1,2\}}}\left(k \Lambda_{\mathbf{b}}\right)\right)=h^{0}\left(k \Lambda_{\mathbf{b}}\right)=1$, it suffices to show that $k \Lambda_{\mathbf{a}}-$ $\delta_{\{0,1\}}$ is not effective, so that $H^{0}\left(\mathcal{O}_{\bar{M}_{0, N+2}}\left(k \Lambda_{\mathbf{a}}-\delta_{\{0,1\}}\right)\right)=0$. To do this, we exhibit a family of irreducible curves such that

(i) For $C$ in the family, $C \cdot\left(k \Lambda_{\mathbf{a}}-\delta_{\{1,2\}}\right)=-1$.

(ii) For a general point of $\bar{M}_{0, N+2}$, some curve in the family passes through the point.

Then the divisor $k \Lambda_{\mathbf{a}}-\delta_{\{1,2\}}$ cannot be effective: a codimension one subvariety with class $k \Lambda_{\mathbf{a}}-\delta_{\{1,2\}}$ must contain each curve $C$ in the family, an absurdity since the curves cover an open subset of $\bar{M}_{0, N+2}$.

We now construct a family of curves satisfying (i) and (ii). Using formulas for classes with respect to the Kapranov basis in index 1, given in Theorem 4.2, we have that

$$
\Lambda_{\mathbf{a}} \sim A H-(A-1) E_{2}-(A-1) E_{N}-E_{J^{0}}-E_{J^{1}}+\cdots,
$$

where $J^{i}:=\{2, \ldots, N\} \backslash\{2, N, N+1+i\}$ for $i=0$ or $i=1$, and $A:=a_{1}+$ $2 m-1$. Other terms contribute to the class, but these are linearly independent and irrelevant.

The significance of the terms $E_{J^{i}}$ is that under $\psi_{1}, E_{J^{i}}$ is mapped to a codimension 2 span not containing $p_{2}$ and not containing $p_{N}$ (these points correspond to $E_{2}$ and $E_{N}$ under the index 1 Kapranov map). Given a point $y \in \mathbb{P}^{N-1}$ not lying on the lined spanned by $p_{2}$ and $p_{N}$, consider the two-plane $T_{y}=\left\langle y, p_{2}, p_{N}\right\rangle$. If $y \notin \psi_{1}\left(E_{J^{i}}\right), i=1,2$, then there exist a unique $x_{i} \in \psi_{1}\left(E_{J^{i}}\right) \cap T_{y}$.

ClAIM 7.3. For a general point $y \in \mathbb{P}^{N-1}$, the points $x_{i}, p_{j}$, and $y$ as constructed before lie in general position in $T_{y} \simeq \mathbb{P}^{2}$.

Given this, for general $y$, we have a unique irreducible conic $C_{y} \subset T_{y}$ passing through all five points, and $C_{y}$ has class $2 h+e_{2}+e_{N}+e_{J^{1}}+e_{J^{0}}$ with respect to the dual of the index 1 Kapranov basis. Pairing with $\Lambda_{\mathbf{a}}$, we see that

$$
C_{y} \cdot \Lambda_{\mathbf{a}}=2 A-2(A-1)-2=0 .
$$

Since $C_{y} \cdot \delta_{\{1,2\}}=1$, it follows that

$$
C_{y} \cdot\left(k \Lambda_{\mathbf{a}}-\delta_{\{1,2\}}\right)=-1 .
$$


Since $C_{y}$ can be defined for a general point $y$ in $\mathbb{P}^{N-1}$, taking the proper transforms of $C_{y}$ under the $\psi_{1}$ gives a family of curves in $\bar{M}_{0, N}$ satisfying (i) and (ii).

To prove Claim 7.3, we first show that $x_{0}, x_{1}$, and $p_{i}$ are noncollinear for general $y$ and $i \in\{2, N\}$. Consider projection from $p_{2}$ to $\mathbb{P}^{N-2}$. Let $L$ be the image of $T_{y}$, and $M_{i}$ be the image of the linear span $\left\langle p_{j}\right\rangle_{j \in J^{i}}$. Each $M_{i}$ is of codimension 1 , and $L$ is of dimension 1. Hence, $L \cap M_{i}$ consists of a single point for each $i$. If $x_{0}, x_{1}$, and $p_{2}$ are collinear, then $L \cap M_{0}=L \cap M_{1}$. Composing our first projection with a second projection from $p_{N}$ to obtain a map $\pi: \mathbb{P}^{N-1} \rightarrow$ $\mathbb{P}^{N-3}$, we see that $M_{0} \cap L=M_{1} \cap L$ occurs if and only if $\pi(y)$ lies in the image of $\left\langle p_{j}\right\rangle_{j \in J^{0} \cap J^{1}}$ under $\pi$, which is of codimension 1 in $\mathbb{P}^{N-3}$.

Note that $p_{2}, p_{N}, y$ and $p_{2}, p_{N}, x_{i}$ are noncollinear for general $y$. So, to conclude that the points are in general position, it suffices to verify that $y, x_{i}$, $p_{j}$ are noncollinear for each $i \in\{2, N\}$ and $j \in\{0,1\}$. By symmetry we may check only for $i=2, j=0$. Note that $x_{0}, y$, and $p_{2}$ are collinear if and only if the image of $y$ under projection from $p_{2}$ lies in the image of $\psi_{1}\left(E_{J^{0}}\right)$ under projection. Since $2 \notin J^{0}$, this image is of codimension 1 , and a general point $y$ is not contained.

REMARK 7.4. While rigidity is not known to imply the extremality on $\bar{M}_{0, n}$, we are unaware of any examples of rigid, nonextremal divisors on the space in question. Rigidity of a divisor class $D$ implies that $D$ cannot be written as a nonnegative linear combination of effective divisors with rational coefficients; for extremality, we must have that $D$ cannot be written as a nonnegative linear combination of pseudo-effective divisors.

We now turn our attention to a class of Chen-Coskun divisors that can be written as linear combinations of other effective divisors and so are nonrigid and nonextremal. Consider an $n$-tuple of nonzero integers $\mathbf{a}=\left(a_{1}, \ldots, a_{n}\right)$ with $\sum_{i} a_{i}=0$; assume that $a_{1}>0$ and $a_{n}<0$. Define $\tilde{\mathbf{a}}=\left(a_{1}+1, a_{2}, \ldots, a_{n-1}, a_{n}-1\right) . \Lambda_{\mathbf{a}}$ and $\Lambda_{\tilde{\mathbf{a}}}$ both defined Chen-Coskun divisors on $\bar{M}_{0, n+2}$. We compare their classes with respect to the Kapranov basis in index 1. From class formulas we see that

$$
\Lambda_{\tilde{\mathbf{a}}}=\left(\sum_{i=2}^{n}\left|a_{i}\right|\right) H+\cdots
$$

and

$$
\Lambda_{\mathbf{a}}=\left(\left(\sum_{i=2}^{n}\left|a_{i}\right|\right)-1\right) H+\cdots
$$

Furthermore, we claim that the coefficient of $E_{J}$ in the class of $\Lambda_{\mathbf{a}}$ and $\Lambda_{\tilde{\mathbf{a}}}$ will be the same whenever $n \in J$ : given an $n$-tuple $\mathbf{b}=\left(b_{i}\right)$ satisfying the appropriate conditions, the coefficient of $E_{J}$ in the class of $\Lambda_{\mathbf{b}}$ is a function of $b_{i}$ for $i \notin J$ and $i \neq 1$. Since $a_{i}$ and $\tilde{a}_{i}$ agree for $i \neq 1$ and $i \neq n$, the claim follows. 
With these preliminary observations, we can conclude that if $\Lambda_{\mathbf{a}}=d H-$ $\sum_{I} m_{I} E_{I}$ and $\Lambda_{\tilde{\mathbf{a}}}=e H-\sum_{I} n_{I} E_{I}$, then

$$
\Lambda_{\tilde{\mathbf{a}}}-\Lambda_{\mathbf{a}}=H-\sum_{n \notin I}\left(n_{I}-m_{I}\right) E_{I} .
$$

Applying Theorem 4.10, we see that, for $n, n+1, n+2 \notin J$,

$$
n_{I}-m_{I}=\left|a_{n}-1\right|-\left|a_{n}\right|=1,
$$

and for $n \notin J,|\{n+1, n+2 \cap J\}|=1$, we have that

$$
=\min \left\{\sum_{\substack{i \notin J \cup\{n\} \\ a_{i} \leq 0}}\left|a_{i}\right|+\left|a_{n}\right|+1, \sum_{\substack{i \notin J \\ a_{i} \geq 0}}\left|a_{i}\right|\right\}-\min \left\{\sum_{\substack{i \notin J \\ a_{i} \leq 0}}\left|a_{i}\right|, \sum_{\substack{i \notin J \\ a_{i} \geq 0}}\left|a_{i}\right|\right\} .
$$

If $\left|a_{n}\right| \geq \sum_{a_{i} \geq 0}\left|a_{i}\right|$, then both minima are equal to the positive sum, and the coefficient of $E_{J}$ is zero. From this we easily obtain the following:

THEOREM 7.5. For $\mathbf{a}=\left(a_{1}, \ldots, a_{n}\right)$ with nonzero $a_{i}, \sum_{i} a_{i}=0, a_{1}>0$, and $a_{n}<0$, define $\tilde{\mathbf{a}}=\left(a_{1}+1, a_{2}, \ldots, a_{n-1}, a_{n}-1\right)$. If

$$
\left|a_{n}\right| \geq \sum_{\substack{a_{i} \geq 0 \\ i \geq 2}}\left|a_{i}\right|,
$$

then $\Lambda_{\tilde{\mathbf{a}}}=\Lambda_{\mathbf{a}}+D$, where $D$ is an effective sum of boundary divisor classes. In particular, $\Lambda_{\tilde{a}}$ is not extremal.

Proof. By the previous discussion,

$$
\Lambda_{\tilde{\mathbf{a}}}=\Lambda_{\mathbf{a}}+H-\sum_{\substack{n \notin J \\ n+1, n+2 \notin J}} E_{J} .
$$

However,

$$
H-\sum_{\substack{n \notin J \\ n+1, n+2 \notin J}} E_{J}=\delta_{n+1, n+2}+\sum_{\substack{n+1, n+2 \in J \\ n \in J}} E_{J},
$$

which is effective.

Example 7.6. If $k \geq d m$ for $k, d, m$ positive integers with $\operatorname{gcd}(k, m)=1$, then let $\mathbf{a}(k, d, m)$ be the $2 d+2$-tuple $(k, m, m, m, \ldots,-m,-m,-m, \ldots,-k)$. Then $\Lambda_{\mathbf{a}(k, d, m)} \subset \bar{M}_{0,2 d+4}$ is not extremal. Indeed, this follows immediately from Theorem 7.5 since the hypothesis that $\left|a_{2 d+2}\right|>1$ and

$$
\left|a_{2 d+2}\right| \geq \sum_{\substack{i \geq 2 \\ a_{i}>0}}\left|a_{i}\right|
$$

is satisfied. 
REMARK 7.7. The hypothesis of Theorem 7.5 are not necessary for nonextremality. The divisors $L_{k}$ from Example 4.8 give another class of nonextremal ChenCoskun divisors, but these do not satisfy the hypotheses of Theorem 7.5. A proof of this is roughly as follows: it was observed in Example 4.8 that for appropriate choice of Kapranov basis, there exist indices $i$ and $j$ such that $E_{I}$ has zero coefficient in the class of $L_{k}$ whenever $i \in I$ or $j \in I$. Hence, these divisors can be realized as pull-backs of nonboundary (and hence nonextremal) divisors from appropriate Losev-Manin spaces [LM]. The argument generalizes to any ChenCoskun divisor corresponding to an $n$-tuple with only one positive entry.

We now return to the implications of Theorem 7.5. For fixed $d$, all but finitely many divisors $\Lambda_{\mathbf{a}}$ on $\bar{M}_{0,2 d+2}$ for $a$ of the form $(k, m, m, m, \ldots,-k,-m,-m$, $-m, \ldots)$ are nonextremal. This is in contrast with the results of [CC], where divisors on $\bar{M}_{1,4}$ arising from 4-tuples of the form $(k, m,-k,-m)$ were shown to be extremal and yielded the result that $\overline{\operatorname{Eff}}\left(\bar{M}_{1, n}\right)$ is not finitely generated. These particular $n$-tuples cannot yield distinct extremal divisors on $\bar{M}_{0,6}$ since $\overline{\operatorname{Eff}}\left(\bar{M}_{0,6}\right)$ is generated by the spherical bipyramid divisor together with boundary classes. In particular, the divisors on $\bar{M}_{0,6}$ corresponding to $(k, m,-k,-m)$ are extremal if and only if $k=m=1$. However, a natural question is whether many extremal rays might arise from "analogous" $n$-tuples with $n$ sufficiently large. The above discussion rules out certain generalizations.

Moreover, Theorem 7.5 provides an obstruction to the construction of "large families" of extremal Chen-Coskun divisors on $\bar{M}_{0, n}$ for $n$ fixed. More precisely, obvious schemes for constructing infinite families of Chen-Coskun divisors can provide only finitely many extremal examples. For instance, fixing all but two indices of a given $n$-tuple and varying these can yield an extremal divisor for only finitely many choices since, after some point, one of the variable entries will become large enough in absolute value so that Theorem 7.5 guarantees that the divisor is nonextremal. However, more innovative approaches to varying $n$-tuples coupled with finer analysis of combinatorial constraints might yield interesting results.

ACKNOWLEDGMents. I am grateful to Jenia Tevelev: this paper began as a summer 2013 REU project under his instruction at the University of Massachusetts Amherst, and his guidance was instrumental in its creation. I also want to thank Anna Kazanova, Tassos Vogiannou, and Julie Rana for helping me to learn Macaulay2 and debug programs, Ana-Maria Castravet for discussion during the 2013 Young Mathematicians Conference at the Ohio State University, Stephen Coughlan and Eduardo Cattani for feedback on earlier drafts, and Angelo Felice Lopez for bringing to my attention arguments in need of tightening. I would also like thank the referee for their suggestions, which I found invaluable.

\section{References}

[CC] D. Chen and I. Coskun, Extremal effective divisors on the moduli space of n-pointed genus one curves, Math. Ann. 359 (2014), no. 3-4, 891-908. 
[CT] A. Castravet and E. Tevelev, Hypertrees, projections, and moduli of stable rational curves, J. Reine Angew. Math. 675 (2013), 121-180.

[FM] W. Fulton and R. MacPherson, A compactification of configuration spaces, Ann. of Math. 139 (1994), 183-225.

[K] M. Kapranov, Chow quotients of Grassmanians I, I. M. Gelfand seminar, Adv. Soviet Math., 16, Part 2, pp. 29-110, Amer. Math. Soc., Providence, 1993.

[K1] - Veronese curves and Grothendieck-Knudsen moduli space $\bar{M}_{0, n}$, J. Algebraic Geom. 2 (1993), 239-262.

[KT] S. Keel and E. Tevelev, Equations of $\bar{M}_{0, n}$, Internat. J. Math. 20 (2009), no. 9, $1-26$.

[LM] A. Losev and Y. Manin, New moduli spaces of pointed curves and pencils of flat connections, Michigan Math. J. 48 (2000), 443-472.

[Op] M. Opie, Hypertree divisor classes, 〈www.math.umass.edu/ tevelev/HT_database/ database $\rangle$.

[Sch] I. Scheidwasser, Hypergraph curves, Honors thesis at the, University of Massachusetts Amherst. 〈www.math.umass.edu/ tevelev〉.

Harvard Math Department

1 Oxford $\mathrm{St}$

Cambridge, MA 021438

USA

opie@math.harvard.edu 